\title{
Well-Posedness of a Navier-Stokes/Mean Curvature Flow system
}

\author{
Helmut Abels and Maximilian Moser \\ Fakultät für Mathematik, Universität Regensburg, Universitätsstraße 31, D-93053 \\ Regensburg, Germany \\ E-mail: Helmut.Abels@mathematik.uni-regensburg.de, \\ Maximilian1.Moser@mathematik.uni-regensburg.de
}

June 1, 2017

\begin{abstract}
We consider a two-phase flow of two incompressible, viscous and immiscible fluids which are separated by a sharp interface in the case of a simple phase transition. In this model the interface is no longer material and its evolution is governed by a convective mean curvature flow equation, which is coupled to a two-phase Navier-Stokes equation with Young-Laplace law. The problem arises as a sharp interface limit of a diffuse interface model, which consists of a Navier-Stokes system coupled with an AllenCahn equation. We prove existence of strong solutions for sufficiently small times and regular initial data.
\end{abstract}

Mathematics Subject Classification (2000):

Primary: 35R35; Secondary 35Q30, 76D27, 76D45, 76T99.

Key words: Two-phase flow, Navier-Stokes system, free boundary problems, mean curvature flow.

\section{Introduction}

We consider the flow of two immiscible, incompressible Newtonian fluids with phase transition in a bounded, smooth domain $\Omega \subseteq \mathbb{R}^{n}, n=2,3$. At time $t \in(0, T)$ the fluids fill domains $\Omega^{ \pm}(t)$ separated by the interface $\Gamma(t):=\partial \Omega^{+}(t)$. For simplicity we set the densities to one. Moreover, we assume that the fluids have constant viscosities $\mu^{ \pm}>0$. Then the stress tensor has the form $T(v, p)=2 \mu^{ \pm} \operatorname{sym}(\nabla v)-p I$, where $\operatorname{sym}(\nabla v):=\frac{1}{2}\left(\nabla v+\nabla v^{\top}\right), v: \Omega \times(0, T) \rightarrow \mathbb{R}^{n}$ is the velocity and $p: \Omega \times(0, T) \rightarrow \mathbb{R}$ is the pressure in Eulerian coordinates.

To formulate the model, we need some notation. We denote by $\nu_{\Gamma(t)}$ the outer unit normal to $\Gamma(t)=\partial \Omega^{+}(t)$ and by $V_{\Gamma(t)}, H_{\Gamma(t)}$ the normal velocity and mean curvature (for convenience the sum of the principal curvatures) with respect to $\nu_{\Gamma(t)}$. Furthermore the jump of a quantity $f$ defined on $\Omega^{ \pm}(t)$ across $\Gamma(t)$ (with respect to $\nu_{\Gamma(t)}$ ) is defined as

$$
\llbracket f \rrbracket(x):=\lim _{r \rightarrow 0} f\left(x+r \nu_{\Gamma(t)}(x)\right)-f\left(x-r \nu_{\Gamma(t)}(x)\right)
$$

for all $x \in \Gamma(t)$. 


\section{INTRODUCTION}

As model we consider the following Navier-Stokes/mean curvature flow system

$$
\begin{aligned}
\partial_{t} v+v \cdot \nabla v-\mu^{ \pm} \Delta v+\nabla p & =0 & & \text { in } \Omega^{ \pm}(t), t \in(0, T), \\
\operatorname{div} v & =0 & & \text { in } \Omega^{ \pm}(t), t \in(0, T), \\
-\llbracket T(v, p) \rrbracket \nu_{\Gamma(t)} & =\sigma H_{\Gamma(t)} \nu_{\Gamma(t)} & & \text { on } \Gamma(t), t \in(0, T), \\
\llbracket v \rrbracket & =0 & & \text { on } \Gamma(t), t \in(0, T), \\
\left.v\right|_{\partial \Omega} & =0 & & \text { on } \partial \Omega \times(0, T), \\
\left.v\right|_{t=0} & =v_{0} & & \text { in } \Omega, \\
V_{\Gamma(t)}-\left.\nu_{\Gamma(t)} \cdot v\right|_{\Gamma(t)} & =H_{\Gamma(t)} & & \text { on } \Gamma(t), t \in(0, T), \\
\Gamma(0) & =\Gamma_{0}, & &
\end{aligned}
$$

where $\sigma>0$ is a surface tension constant and $\Gamma_{0}$ is the initial interface. Here the velocity $v$, the pressure $p$ and the family of interfaces $\{\Gamma(t)\}_{t \in(0, T)}$ are to be determined. Equations (1.1)-(1.2) describe the (local) mass and momentum conservation of the fluids and (1.3) is the balance of forces at the interface, see Landau and Lifshitz [15], §61. Moreover we require continuity of $v$ on $\Gamma(t)$ in (1.4) and the no-slip condition for $v$ on $\partial \Omega$ in (1.5). (1.6) and (1.8) are the initial conditions for $v$ and the family of hypersurfaces $\{\Gamma(t)\}_{t \in(0, T)}$, respectively. Equation (1.7) without the curvature term $H_{\Gamma(t)}$ means that the interface is just transported by the fluids. Then (1.1)-(1.8) reduces to the classical two-phase Navier-Stokes system with surface tension, which was studied e.g. by Köhne, Prüss and Wilke [14]. For this system the total mass of each fluid is preserved since the densities are constant and the enclosed volume of $\Gamma(t)$ stays the same. Formally this follows from (references in) Escher and Simonett [7], p. 623, the divergence theorem and (1.2):

$$
\frac{d}{d t}\left|\Omega^{+}(t)\right|=\int_{\Gamma(t)} V_{\Gamma(t)} d \sigma=\int_{\Gamma(t)} v \cdot \nu_{\Gamma(t)} d \sigma=\int_{\Omega^{+}(t)} \operatorname{div} v d x=0 .
$$

The additional curvature term $H_{\Gamma(t)}$ on the right hand side in (1.7) allows for a change of total masses and couples the Navier-Stokes system to the mean curvature flow

$$
V_{\Gamma(t)}=H_{\Gamma(t)} \quad \text { for } t>0 .
$$

Therefore we call (1.1)-(1.8) the Navier-Stokes/mean curvature flow system. The system is obtained as a sharp interface limit of a Navier-Stokes-Allen-Cahn system. Another motivation is the regularizing effect of the curvature term in (1.7). But we can also view it as a simple model for a two-phase flow with phase transition. In the case that the Navier-Stokes equations are modified for shear thickening non-Newtonian fluid of powerlaw type Liu, Sato, and Tonegawa in [16] have shown existence of weak solutions. In the present contribution we prove local existence of strong solutions to (1.1)-(1.8) in an $L^{p}$-setting. The result is part of the second author's Master thesis, which was supervised by the first author.

We apply a similar strategy as A. and Wilke [2], who study local well-posedness and qualitative behaviour of solutions for a two-phase Navier-Stokes-Mullins-Sekerka system. The equations are transformed with a variant $\tilde{\Theta}_{h}$ of the Hanzawa transform to a reference surface $\Sigma$ and disjoint domains $\Omega^{ \pm}$, where $\Omega=\Omega^{+} \cup \Omega^{-} \cup \Sigma$ is similar as above. Here $h: \Sigma \times(0, T) \rightarrow \mathbb{R}$ is the "height function" of $\{\Gamma(t)\}_{t \in(0, T)}$ with respect to 


\section{Preliminaries}

$\Sigma$. The coupling with the Navier-Stokes part in (1.7) is of lower order. Therefore we solve the Navier-Stokes-part (1.1)-(1.6) for a given appropriate family of hypersurfaces $\{\Gamma(t)\}_{t \in(0, T)}$ or equivalently for a given suitable time-dependent height function $h$. Then we insert the obtained velocity field $u(h)$ into equation (1.7) and (1.7)-(1.8) turns into an abstract evolution equation for $h$. The latter is solved by using the theory of maximal $L^{p}$-regularity.

The paper is organized as follows. In Section 2 we describe the transformation to a fixed reference hypersurface (and related domains) and analyse the occurring terms, especially the transformed mean curvature. In Section 3 we consider the Navier-Stokes part for a given family of hypersurfaces $\left\{\Gamma_{h}(t)\right\}_{t \in(0, T)}$ and in Section 4 we show the local well-posedness for the transformed system. Definitions and some properties of the used Banach-space valued function spaces are summarized in Appendix $\mathrm{A}$. In Appendix B we prove a maximal regularity result for a two-phase Stokes system which is needed for Section 3 .

We always assume $\Omega \subseteq \mathbb{R}^{n}, n=2,3$, to be a bounded, connected and smooth domain and throughout let $\Sigma \subseteq \Omega$ be a compact, connected and smooth hypersurface, that separates $\Omega$ in two disjoint, connected domains $\Omega^{ \pm}$with $\Sigma=\partial \Omega^{+}$and outer unit normal $\nu_{\Sigma}$.

Acknowledgements: The second author is grateful to Mathias Wilke for many helpful discussions and his lecture series on "maximal regularity".

\section{Preliminaries}

\subsection{Notation and Scalar-Valued Function Spaces}

Let $\mathbb{R}_{+}:=[0, \infty)$ and $\mathbb{R}_{+}^{n}:=\mathbb{R}^{n-1} \times(0, \infty)$ for $n \in \mathbb{N}, n \geq 2$. The Euclidean norm in $\mathbb{R}^{n}$ is denoted by |.|. For matrices $A, B \in \mathbb{R}^{n \times n}$ let $A: B$ be the matrix-product and $|A|:=\sqrt{A: A}$ be the induced norm. The set of invertible matrices in $\mathbb{R}^{n \times n}$ is $G L(n, \mathbb{R})$. For $A \in G L(n, \mathbb{R})$ we set $A^{-\top}:=\left(A^{-1}\right)^{\top}$.

For metric spaces $X$ denote by $B_{X}(x, r)$ the ball with radius $r>0$ around $x \in X$. For normed spaces $X, Y$ over $\mathbb{K}=\mathbb{R}$ or $\mathbb{C}$ the set of bounded, linear operators $T: X \rightarrow Y$ is $\mathcal{L}(X, Y)$ and $\mathcal{L}(X):=\mathcal{L}(X, X)$. If $X, Y$ are additionally complete and $U \subseteq X$ is open, for $k \in \mathbb{N}$ the set of all $k$-times continuously differentiable functions $f: U \rightarrow Y$ is denoted by $C^{k}(U ; Y)$. The set of all bounded $f \in C^{k}(U ; Y)$ with bounded derivatives is $B C^{k}(U ; Y)$ with usual norm.

If $\left(X_{0}, X_{1}\right)$ is admissible and $\theta \in(0,1), 1 \leq p \leq \infty$, we denote by $\left(X_{0}, X_{1}\right)_{\theta, p}$ the real interpolation spaces and with $\|\cdot\|_{\left(X_{0}, X_{1}\right)_{\theta, p}} \equiv\|\cdot\|_{\theta, p}$ the norm, see Lunardi [17], Bergh and Löfström [5] and Triebel [22].

Let $\Omega \subseteq \mathbb{R}^{n}, n \in \mathbb{N}$, be open and nonempty. Spaces of continuous and continuously differentiable functions $f: \bar{\Omega} \rightarrow \mathbb{R}$ are defined as usual. Now let $1 \leq p \leq \infty$ and $k \in \mathbb{N}$. Then the Lebesgue and Sobolev spaces are denoted by $L^{p}(\Omega)=: W_{p}^{0}(\Omega)$ and $W_{p}^{k}(\Omega)$, respectively. Moreover, we set

$$
W_{p, 0}^{k}(\Omega):={\overline{C_{0}^{\infty}(\Omega)}}^{W_{p}^{k}(\Omega)} \quad \text { and } \quad W_{p}^{-k}(\Omega):=W_{p^{\prime}, 0}^{k}(\Omega)^{\prime},
$$




\section{Preliminaries}

where $p^{\prime}$ is the conjugate exponent to $p$, as well as $L_{(0)}^{p}(\Omega):=\left\{f \in L^{p}(\Omega): \int_{\Omega} f d x=0\right\}$,

$$
W_{p,(0)}^{k}(\Omega):=W_{p}^{k}(\Omega) \cap L_{(0)}^{p}(\Omega) \quad \text { and } \quad W_{p,(0)}^{-k}(\Omega):=W_{p^{\prime},(0)}^{k}(\Omega)^{\prime} .
$$

The closure of the divergence-free $\phi \in C_{0}^{\infty}(\Omega)^{n}$ in $L^{p}(\Omega)^{n}$ is denoted by $L_{\sigma}^{p}(\Omega)$. Besides, we need the Besov spaces $B_{p, q}^{s}\left(\mathbb{R}^{n}\right)$ for $0<s<\infty$ and $1 \leq p, q \leq \infty$. We set $W_{p}^{s}\left(\mathbb{R}^{n}\right):=$ $B_{p, p}^{s}\left(\mathbb{R}^{n}\right)$ for all $s \in(0, \infty) \backslash \mathbb{N}, 1 \leq p \leq \infty$. Respective spaces can also be defined on bounded, smooth domains $\Omega \subseteq \mathbb{R}^{n}$ and, if $n \geq 2$, on their boundaries $\partial \Omega$. For embeddings, interpolation results and trace theorems, compare e.g. Triebel [22], [23].

\subsection{Hanzawa Transformation $\Theta_{h}$}

For the construction of $\Theta_{h}$ we use that there is an $a>0$, such that

$$
X: \Sigma \times(-a, a) \rightarrow \mathbb{R}^{n}:(s, r) \mapsto s+r \nu_{\Sigma}(s)
$$

is a $C^{\infty}$-diffeomorphism onto $\Sigma_{a}:=B_{a}(\Sigma)$. We set $\left(\Pi, d_{\Sigma}\right):=X^{-1}$. Then it holds $\nabla d_{\Sigma}=\nu_{\Sigma} \circ \Pi$. Here $\Pi$ is called projection onto $\Sigma$ and $d_{\Sigma}$ signed distance function. For the proof cf. Hildebrandt [11], Chapter 4.6, Theorems 1-3.

Let $a<\operatorname{dist}(\Sigma, \partial \Omega)$ and $a_{0}<\frac{a}{4}$ fixed, $\chi \in C^{\infty}(\mathbb{R})$ be a cutoff function such that $\left|\chi^{\prime}\right| \leq 4$ and $\chi(s)=1$ for $|s| \leq \frac{1}{3}$, as well as $\chi(s)=0$ for $|s| \geq \frac{2}{3}$. For $h \in C^{2}(\Sigma)$ with $\|h\|_{\infty}<a_{0}$ we define the Hanzawa transformation $\Theta_{h}$ by

$$
\Theta_{h}(x):=x+\chi\left(\frac{d_{\Sigma}(x)}{a}\right) h(\Pi(x)) \nu_{\Sigma}(\Pi(x)) \quad \text { for all } x \in \mathbb{R}^{n} .
$$

Essential properties of $\Theta_{h}$ are listed in

Lemma 2.1 The Hanzawa transformation $\Theta_{h}: \Omega \rightarrow \Omega$ is a $C^{2}$-diffeomorphism and $\Theta_{h}$ is the identity on $\mathbb{R}^{n} \backslash \Sigma_{2 a / 3}$, in particular nearby $\partial \Omega$. Moreover,

$$
\operatorname{det} D \Theta_{h} \geq c>0 \quad \text { and } \quad\left\|D \Theta_{h}\right\|_{\infty}+\left\|D \Theta_{h}^{-1}\right\|_{\infty} \leq C\left(1+\|h\|_{C^{1}(\Sigma)}\right)
$$

with $c, C>0$ independent of $h$.

Proof. The first part can be shown as in Kneisel [12], Lemma 2.2.1 and one also obtains a local representation for $D \Theta_{h}$. The latter yields the estimates by compactness of $\Sigma$.

Remark 2.2 Let $h \in C^{2}(\Sigma)$ such that $\|h\|_{\infty}<a_{0}$. Then $\Gamma_{h}:=\Theta_{h}(\Sigma)$ is a connected and compact $C^{2}$-hypersurface as zero level set of

$$
\phi_{h}: \Sigma_{a} \rightarrow \mathbb{R}: x \mapsto d_{\Sigma}(x)-h(\Pi(x)),
$$

where $\left|\nabla \phi_{h}\right| \geq 1$ as one can show $\left.\left.\nabla \phi_{h}\right|_{x} \cdot \nu_{\Sigma}\right|_{\Pi(x)}=1$ for all $x \in \Sigma_{a}$ similar to Kneisel [12], p. 13. Moreover, $\nu_{\Gamma_{h}}(x):=\nabla \phi_{h}(x) /\left|\nabla \phi_{h}(x)\right|$ is the outer unit normal to $\Gamma_{h}=$ $\partial\left(\Theta_{h}\left(\Omega^{+}\right)\right)$at $x \in \Gamma_{h}$. Furthermore, because of $\phi_{h}\left(\Theta_{h}(x)\right)=d_{\Sigma}(x)$ on $\overline{\Sigma_{a / 3}}$, we get the identity $\left.\nabla \phi_{h}\right|_{\Theta_{h}(x)}=\left.\left.D \Theta_{h}^{-\top}\right|_{x} \nu_{\Sigma}\right|_{\Pi(x)}$ for $x \in \overline{\Sigma_{a / 3}}$ and this implies

$$
\left.\left.\left.D \Theta_{h}^{-\top}\right|_{x} \nu_{\Sigma}\right|_{\Pi(x)} \cdot \nu_{\Sigma}\right|_{\Pi(x)}=1 \quad \text { for all } x \in \overline{\Sigma_{a / 3}} .
$$




\section{Preliminaries}

\subsection{Function Spaces for the Height Function and Modification $\tilde{\Theta}_{h}$ of $\Theta_{h}$}

In the following we specify, in which function spaces we consider height functions $h$. Let $p>n+2$ be fixed. We set

$$
X_{0}:=W_{p}^{1-\frac{1}{p}}(\Sigma) \quad \text { and } \quad X_{1}:=W_{p}^{3-\frac{1}{p}}(\Sigma) .
$$

Since $3-\frac{3}{p}-\frac{n-1}{p}>2$ we have $X_{\gamma}:=\left(X_{0}, X_{1}\right)_{1-\frac{1}{p}, p}=W_{p}^{3-\frac{3}{p}}(\Sigma) \hookrightarrow C^{2}(\Sigma)$. Moreover, we define $\mathbb{E}_{1}(T):=L^{p}\left(0, T ; X_{1}\right) \cap W_{p}^{1}\left(0, T ; X_{0}\right)$. Theorem A.6yields $\mathbb{E}_{1}(T) \hookrightarrow C^{0}\left([0, T] ; X_{\gamma}\right)$. Hence

$$
\Theta_{h}(x, t):=\Theta_{h(., t)}(x) \quad \text { for }(x, t) \in \mathbb{R}^{n} \times[0, T]
$$

is well-defined for $h \in \mathbb{E}_{1}(T) \cap C^{0}([0, T] ; U)$ where $U:=\left\{h \in X_{\gamma}:\|h\|_{\infty}<a_{0}\right\}$. Let $R_{0}>0$ arbitrary and fixed from now on. It suffices to consider height functions in the space

$$
V_{T}:=\left\{h \in \mathbb{E}_{1}(T) \cap C^{0}([0, T] ; U):\|h\|_{\mathbb{E}_{1}(T)}+\|h(0)\|_{X_{\gamma}}<R_{0}\right\} .
$$

The restriction due to the estimate is negligible as we are interested in local wellposedness, but it is needed to show suitable properties of a modification of the Hanzawa transformation $\Theta_{h}$ which we need for technical reasons: if we transform differential operators in space, the term $D \Theta_{h}^{-\top}$ always appears. In order to show appropriate regularity properties in space of $D \Theta_{h}^{-\top}$, it is complicated (but possible) to work with the Hanzawa transformation directly. However, it seems difficult to obtain suitable regularity in time for $D \Theta_{h}^{-\top}$ since derivatives of $h$ in space are present. It is much simpler to replace $h \circ \Pi$ in $\Theta_{h}$ by an extension $E h$ of $h$, where for $\bar{a}:=\frac{3 a}{4}$

$$
E: X_{0}=W_{p}^{1-\frac{1}{p}}(\Sigma) \rightarrow W_{p}^{1}\left(\Sigma_{\bar{a}}\right) \quad \text { with } E \in \mathcal{L}\left(W_{p}^{k-\frac{1}{p}}(\Sigma), W_{p}^{k}\left(\Sigma_{\bar{a}}\right)\right), k=1,2,3
$$

is a suitable extension operator. Then we have $E \in \mathcal{L}\left(X_{\gamma}, \tilde{X}_{\gamma}\right)$, where we have set $\tilde{X}_{\gamma}:=\left(W_{p}^{1}\left(\Sigma_{\bar{a}}\right), W_{p}^{3}\left(\Sigma_{\bar{a}}\right)\right)_{1-\frac{1}{p}, p}$. Here because of $p>n+2$ it holds

$$
\tilde{X}_{\gamma}=W_{p}^{3-\frac{2}{p}}\left(\Sigma_{\bar{a}}\right) \hookrightarrow C^{2}\left(\overline{\Sigma_{\bar{a}}}\right) .
$$

So we define the modification $\tilde{\Theta}_{h}$ of the Hanzawa transformation $\Theta_{h}$ as follows:

Definition 2.3 Let $p>n+2, R_{0}>0$ as above and $T>0$. For $h_{0} \in U$ we set

$$
\tilde{\Theta}_{h}(x):=x+\chi\left(\frac{d_{\Sigma}(x)}{a}\right) E h(x) \nu_{\Sigma}(\Pi(x)) \quad \text { for all } x \in \mathbb{R}^{n}
$$

and for $h \in V_{T}$ let $\tilde{\Theta}_{h}(x, t):=\tilde{\Theta}_{h(., t)}(x)$ for all $(x, t) \in \mathbb{R}^{n} \times[0, T]$.

The following lemma shows that similar properties as for $\Theta_{h}$ also hold for the modification $\tilde{\Theta}_{h}$ if the extension operator $E$ is chosen properly. 


\section{Preliminaries}

Lemma 2.4 Let $\bar{a}=\frac{3 a}{4}$ and $\varepsilon>0$. Then $E$ can be chosen such that for all $h \in X_{\gamma}$

$$
\|h \circ \Pi-E h\|_{C^{1}\left(\overline{\Sigma_{\bar{a}}}\right)} \leq \varepsilon\|h\|_{X_{\gamma}} .
$$

Moreover, for $R>0$ there is an $\varepsilon=\varepsilon(R)>0$ such that with the extension operator $E$ with respect to $\varepsilon$ the following hold: $\tilde{\Theta}_{h}: \Omega \rightarrow \Omega$ is for all $h \in U$ with $\|h\|_{X_{\gamma}} \leq R$ a $C^{2}$-diffeomorphism, the identity on $\mathbb{R}^{n} \backslash \Sigma_{2 a / 3}$ and we have

$$
\operatorname{det} D \tilde{\Theta}_{h} \geq \tilde{c}>0 \quad \text { and }\left.\left.\left.\quad D \tilde{\Theta}_{h}^{-\top}\right|_{x} \nu_{\Sigma}\right|_{\Pi(x)} \cdot \nu_{\Sigma}\right|_{\Pi(x)} \geq \frac{1}{2} \quad \text { for all } x \in \overline{\Sigma_{a / 3}} \text {. }
$$

Remark 2.5 Theorem A.6 implies $\mathbb{E}_{1}(T) \hookrightarrow C^{0}\left([0, T] ; X_{\gamma}\right)$ and the embedding constant is bounded independent of $T>0$ if we add the term $\|h(0)\|_{X_{\gamma}}$ in the $\mathbb{E}_{1}(T)$-norm. Now we can do the following: we choose $R=\overline{R_{0}}>R_{0}$ in Lemma 2.4 (and so $E$ ) such that

$$
h(t) \in U_{0}:=\left\{h_{0} \in U:\left\|h_{0}\right\|_{X_{\gamma}}<\overline{R_{0}}\right\}
$$

holds for all $h \in V_{T}$ and $0 \leq t \leq T$. In particular for $h \in V_{T}$ the last part in Lemma 2.4 is valid for $\tilde{\Theta}_{h}(., t)$ instead of $\tilde{\Theta}_{h}$. Here $0 \leq t \leq T$ and $0<T<\infty$ are arbitrary.

Proof of Lemma 2.4. Similar to A. and Wilke [2] one can construct a new operator $E$ that fulfils (2.4) by scaling $E$ in normal direction of $\Sigma$. Now let $R>0$ arbitrary. First of all, $\tilde{\Theta}_{h}$ is well-defined for all $h \in X_{\gamma}$, a $C^{2}$-mapping and the identity on $\mathbb{R}^{n} \backslash \Sigma_{2 a / 3}$, as the second part is cut off properly. Since $\tilde{\Theta}_{h}=\Theta_{h}$ on $\Sigma$, we can use the properties of $\Theta_{h}$. For all $h \in U$ with $\|h\|_{X_{\gamma}} \leq R$ it holds $\|h \circ \Pi-E h\|_{C^{1}\left(\overline{\Sigma_{\bar{a}}}\right)} \leq \varepsilon R$ due to (2.4) and therefore

$$
\left\|D \Theta_{h}-D \tilde{\Theta}_{h}\right\|_{\infty} \leq\left\|\chi\left(\frac{d_{\Sigma}(.)}{a}\right) \nu_{\Sigma}(\Pi(.))(h \circ \Pi-E h)\right\|_{C^{1}\left(\overline{\Sigma_{\bar{a}}}\right)} \leq C \varepsilon R .
$$

Since $\left\{D \Theta_{h}(x): x \in \mathbb{R}^{n}, h \in U,\|h\|_{X_{\gamma}} \leq R\right\}$ is contained in a compact set $K \subseteq G L(n, \mathbb{R})$, we obtain for $\varepsilon(R)>0$ small that $\operatorname{det} D \tilde{\Theta}_{h} \geq \frac{c}{2}>0$. In particular $D \tilde{\Theta}_{h}$ is invertible and the inverse mapping theorem implies that $\tilde{\Theta}_{h}: \Omega \rightarrow \Omega$ is a $C^{2}$-diffeomorphism if we show bijectivity of $\tilde{\Theta}_{h}: \mathbb{R}^{n} \rightarrow \mathbb{R}^{n}$. This can be done for a suitable choice of $\varepsilon(R)$ as in A. and Wilke [2], p. 49 using Lemma 2.1] and (2.5).

It remains to show the second estimate. Lemma 2.1 and (2.5) yield because of $D \tilde{\Theta}_{h}=$ $D \Theta_{h}\left(I-D \Theta_{h}^{-1}\left(D \Theta_{h}-D \tilde{\Theta}_{h}\right)\right)$ together with a Neumann series argument

$$
\left|D \tilde{\Theta}_{h}^{-\top}(x)-D \Theta_{h}^{-\top}(x)\right| \leq \tilde{C}(R) \varepsilon
$$

for all $x \in \mathbb{R}^{n}$ and $h \in U$ with $\|h\|_{X_{\gamma}} \leq R$ if $\varepsilon=\varepsilon(R)>0$ is sufficiently small. Equation (2.1) in Remark 2.2 implies

$$
\left.\left.\left|D \tilde{\Theta}_{h}^{-\top}\right|{ }_{x} \nu_{\Sigma}\right|_{\Pi(x)} \cdot \nu_{\Sigma}\right|_{\Pi(x)}-\left.\left.\left.1|=|\left(D \tilde{\Theta}_{h}^{-\top}-D \Theta_{h}^{-\top}\right)\right|_{x} \nu_{\Sigma}\right|_{\Pi(x)} \cdot \nu_{\Sigma}\right|_{\Pi(x)} \mid \leq \tilde{C}(R) \varepsilon \leq \frac{1}{2}
$$

for all $x \in \overline{\Sigma_{a / 3}}$ if $\varepsilon(R)>0$ is chosen suitably. 


\section{Preliminaries}

\subsection{The Transformed Equations}

With $\tilde{\Theta}_{h}$ we transform the Navier-Stokes/mean curvature flow system (1.1)-(1.8) to $\Sigma$ and $\Omega^{ \pm}$, respectively. Therefore we fix the height function $h \in V_{T}$ and let $v: \Omega \times(0, T) \rightarrow$ $\mathbb{R}^{n}$ be a vector-valued and $\tilde{p}: \Omega \times(0, T) \rightarrow \mathbb{R}$ be a scalar-valued function. Then we define $u(x, t):=v\left(\tilde{\Theta}_{h}(x, t), t\right)$ and $\tilde{q}(x, t):=\tilde{p}\left(\tilde{\Theta}_{h}(x, t), t\right)$ for $(x, t) \in \Omega \times(0, T)$. Moreover, let

$$
A(h):=D \tilde{\Theta}_{h}^{-\top}, \quad \nabla_{h}:=A(h) \nabla, \quad \nabla_{h} u:=\left(\nabla_{h} u_{k}^{\top}\right)_{k=1}^{n} \text { and } \operatorname{div}_{h} u:=\operatorname{Tr} \nabla_{h} u .
$$

Let $t \in[0, T]$. We set $\Gamma_{h}(t):=\Gamma_{h(., t)}$ and denote the outer unit normal by $\nu_{\Gamma_{h}(t)}$ and by $H_{\Gamma_{h}(t)}$ the corresponding mean curvature. Furthermore, we define

$$
K(h): \Sigma \times[0, T] \rightarrow \mathbb{R}:\left.(x, t) \mapsto H_{\Gamma_{h}(t)}\right|_{\Theta_{h}(x, t)} .
$$

Applying the chain rule to $\phi_{h(., t)} \circ \tilde{\Theta}_{h}(., t)$ with $\phi_{h(., t)}$ as in Remark 2.2 implies

$$
\left.\nu_{h}\right|_{(x, t)}:=\left.\nu_{\Gamma_{h}(t)}\right|_{\tilde{\Theta}_{h}(x, t)}=\frac{\left.\left.A(h)\right|_{(x, t)} \nu_{\Sigma}\right|_{x}}{\left.|A(h)|_{(x, t)} \nu_{\Sigma}\right|_{x} \mid} \quad \text { for all }(x, t) \in \Sigma \times(0, T) .
$$

Moreover, we have $\left.V_{\Gamma_{h}(t)}\right|_{\tilde{\Theta}_{h}(x, t)}=\left.\partial_{t}\left(\Theta_{h}(x, t)\right) \cdot \nu_{\Gamma_{h}(t)}\right|_{\Theta_{h}(x, t)}=\left.\left.\partial_{t} h(x, t) \nu_{\Sigma}\right|_{x} \cdot \nu_{h}\right|_{(x, t)}$ for the normal velocity. Since $\tilde{\Theta}_{h}$ is the identity in a neighbourhood of $\partial \Omega$, the no-slip condition on $v$ is retained. Therefore (1.1)-(1.8) becomes

$$
\begin{aligned}
\partial_{t} u+u \cdot \nabla_{h} u-\mu^{ \pm} \operatorname{div}_{h} \nabla_{h} u+\nabla_{h} \tilde{q} & =\nabla_{h} u \partial_{t} \tilde{\Theta}_{h} & & \text { in } \Omega^{ \pm} \times(0, T), \\
\operatorname{div}_{h} u & =0 & & \text { in } \Omega^{ \pm} \times(0, T), \\
-\llbracket T_{h}(u, \tilde{q}) \rrbracket \nu_{h} & =\sigma K(h) \nu_{h} & & \text { on } \Sigma \times(0, T), \\
\llbracket u \rrbracket & =0 & & \text { on } \Sigma \times(0, T), \\
\left.u\right|_{\partial \Omega} & =0 & & \text { on } \partial \Omega \times(0, T), \\
\left.u\right|_{t=0} & =v_{0} \circ \tilde{\Theta}_{h_{0}} & & \text { in } \Omega, \\
\partial_{t} h-\left.a_{1}(h) \cdot u\right|_{\Sigma} & =a_{2}(h) K(h) & & \text { on } \Sigma \times(0, T), \\
\left.h\right|_{t=0} & =h_{0} & & \text { on } \Sigma,
\end{aligned}
$$

where we set $T_{h}(u, \tilde{q}):=2 \mu^{ \pm} \operatorname{sym}\left(\nabla_{h} u\right)-\tilde{q} I$ as well as

$$
a_{1}(h):=\frac{A(h) \nu_{\Sigma}}{A(h) \nu_{\Sigma} \cdot \nu_{\Sigma}} \quad \text { and } \quad a_{2}(h):=\frac{\left|A(h) \nu_{\Sigma}\right|}{A(h) \nu_{\Sigma} \cdot \nu_{\Sigma}}
$$

Moreover $u \cdot \nabla_{h} u \equiv\left(u \cdot \nabla_{h}\right) u$ and $\operatorname{div}_{h}$ is applied row-wise to $\nabla_{h} u$ in (2.6). For this system we show local well-posedness. As a preparation, in the next two Subsections 2.5 and 2.6 we show properties of $\tilde{\Theta}_{h}, A(h)=D \tilde{\Theta}_{h}^{-\top}$ and of the transformed mean curvature $K(h)$, respectively.

\subsection{Properties of $\tilde{\Theta}_{h}$ and $D \tilde{\Theta}_{h}^{-\top}$}

For later use we must know the regularity of $\tilde{\Theta}_{h}$ and $A(h)=D \tilde{\Theta}_{h}^{-\top}$. This is provided by 


\section{Preliminaries}

Lemma 2.6 Let $U_{0}$ as in Remark 2.5 and $0<T \leq T_{0}$. Then $\tilde{\Theta}$. $\in B C^{1}\left(U_{0} ; C^{2}(\bar{\Omega})^{n}\right)$, $A \in B C^{1}\left(U_{0} ; C^{1}(\bar{\Omega})^{n \times n}\right)$ and $\tilde{\Theta} . \in B C^{1}\left(V_{T} ; \tilde{X}_{T}\right), A \in B C^{1}\left(V_{T} ; X_{T}\right)$, where

$$
\begin{aligned}
\tilde{X}_{T} & :=C^{\tau}\left([0, T] ; C^{2}(\bar{\Omega})^{n}\right) \times C^{\frac{1}{2}+\tau}\left([0, T] ; C^{1}(\bar{\Omega})^{n}\right) \cap W_{p}^{1}\left(0, T ; W_{p}^{1}(\Omega)^{n}\right), \\
X_{T} & :=C^{\tau}\left([0, T] ; C^{1}(\bar{\Omega})^{n \times n}\right) \times C^{\frac{1}{2}+\tau}\left([0, T] ; C^{0}(\bar{\Omega})^{n \times n}\right) \cap W_{p}^{1}\left(0, T ; L^{p}(\Omega)^{n \times n}\right)
\end{aligned}
$$

for some $\tau>0$. In the time-dependent case the mappings and their derivatives are bounded by a $C>0$ independent of $0<T \leq T_{0}$ if we add $\|h(0)\|_{X_{\gamma}}$ in the $\mathbb{E}_{1}(T)$-norm.

Proof. Let $h \in U_{0}$ or $h \in V_{T}$. First, we show the properties of $\tilde{\Theta}_{h}$. It holds

$$
\tilde{\Theta}_{h}=\mathrm{id}+\phi E h \quad \text { with } \phi:=\chi\left(d_{\Sigma}(.) / a\right) \nu_{\Sigma} \circ \Pi \in C_{0}^{\infty}\left(\Sigma_{3 a / 4}\right)^{n} .
$$

Here $E$ is the extension operator from Remark 2.5 that induces $E \in \mathcal{L}\left(X_{\gamma}, \tilde{X}_{\gamma}\right)$ with $\tilde{X}_{\gamma} \hookrightarrow C^{2}\left(\overline{\Sigma_{\bar{a}}}\right)$ by $(2.3)$ and $E \in \mathcal{L}\left(\mathbb{E}_{1}(T), \tilde{\mathbb{E}}_{1}(T)\right)$ with

$$
\tilde{\mathbb{E}}_{1}(T):=L^{p}\left(0, T ; W_{p}^{3}\left(\Sigma_{\bar{a}}\right)\right) \cap W_{p}^{1}\left(0, T ; W_{p}^{1}\left(\Sigma_{\bar{a}}\right)\right),
$$

where the operator norm is bounded by a $C>0$ independent of $T>0$. We need suitable embeddings for $\tilde{\mathbb{E}}_{1}(T)$ : Theorem A.7 yields for $0<\theta<1-\frac{1}{p}$

$$
\tilde{\mathbb{E}}_{1}(T) \hookrightarrow C^{1-\theta-\frac{1}{p}}\left([0, T] ; B_{p, p}^{1+2 \theta}\left(\Sigma_{\bar{a}}\right)\right)
$$

and the embedding constant is bounded independent of $T>0$ if we add the term $\|\tilde{h}(0)\|_{\tilde{X}_{\gamma}}$ in the $\tilde{\mathbb{E}}_{1}(T)$-norm. For $\theta=\frac{n}{2 p}+\frac{k-1}{2}+\delta, k=1,2$ and $\delta>0$ small we obtain because of $p>n+2$

$$
1+2 \theta-\frac{n}{p}=1+k-1+2 \delta>k \quad \text { and } \quad 1-\frac{1}{p}-\theta=1-\frac{n+2}{2 p}-\frac{k-1}{2}-\delta>1-\frac{k}{2} .
$$

Embedding theorems and $(2.15)$ imply $\tilde{\mathbb{E}}_{1}(T) \hookrightarrow C^{\tau}\left([0, T] ; C^{2}\left(\overline{\Sigma_{\bar{a}}}\right)\right) \cap C^{\frac{1}{2}+\tau}\left([0, T] ; C^{1}\left(\overline{\Sigma_{\bar{a}}}\right)\right)$ for some $\tau>0$. Since $\tilde{\Theta}_{h}$ is affine linear in $h$, we get the properties of $\tilde{\Theta}_{h}$.

Now we deduce the assertions for $A(h)=D \tilde{\Theta}_{h}^{-\top}$. The first part yields analoguous properties for $D \tilde{\Theta}_{h}$. Moreover, the space $X_{T}$ is an algebra with pointwise multiplication and a product estimate holds because this is valid for the Hölder spaces and we also have Lemma A.9. Additionally, Lemma 2.4 implies $\operatorname{det} D \tilde{\Theta}_{h} \geq c>0$ for all $h \in U_{0}$ and $h \in V_{T}$, respectively. The inverse formula, Lemma A.3 and Lemma A.9 give

$$
A(h) \in C^{1}(\bar{\Omega})^{n \times n} \text { for all } h \in U_{0} \quad \text { and } \quad A(h) \in X_{T} \text { for all } h \in V_{T} .
$$

Furthermore, the mappings are bounded and in the time-dependent case bounded by a $C>0$ independent of $0<T \leq T_{0}$ if we add $\|h(0)\|_{X_{\gamma}}$ in the $\mathbb{E}_{1}(T)$-norm. The implicit function theorem applied to

$$
G: Z \times X \rightarrow X:(h, B) \mapsto D \tilde{\Theta}_{h}^{\top} B-I
$$

at $(h, A(h))$ for $(Z, X)=\left(U_{0}, C^{1}(\bar{\Omega})^{n \times n}\right)$ or $\left(V_{T}, X_{T}\right)$ implies $A \in C^{1}\left(U_{0} ; C^{1}(\bar{\Omega})^{n \times n}\right)$ and $A \in C^{1}\left(V_{T} ; X_{T}\right)$, respectively, with

$$
\frac{d}{d h} A(h)(.)=-A(h) \frac{d}{d h}\left(D \tilde{\Theta}_{h}^{\top}\right)(.) A(h) .
$$

From the properties of $D \tilde{\Theta}_{h}$ and (2.16) the claim follows.

As a consequence we obtain 


\section{Preliminaries}

Corollary 2.7 Let $U_{0}$ be as in Remark 2.5, $0<T \leq T_{0}$ and $\tau>0$ as in Lemma 2.6. Then $\nu_{h}, a_{1}(h)$ and $a_{2}(h)$ as in Subsection 2.4 are well-defined for $h \in U_{0}$ and $h \in V_{T}$, respectively, and have the regularity

$$
B C^{1}\left(U_{0} ; C^{1}(\Sigma)\right) \quad \text { and } \quad B C^{1}\left(V_{T} ; C^{\tau}\left([0, T] ; C^{1}(\Sigma)\right) \cap C^{\frac{1}{2}+\tau}\left([0, T] ; C^{0}(\Sigma)\right)\right) .
$$

In the time-dependent case the mappings and their derivatives are bounded by a $C>0$ independent of $0<T \leq T_{0}$ if we add the term $\|h(0)\|_{X_{\gamma}}$ in the $\mathbb{E}_{1}(T)$-norm.

Proof. Lemma 2.4 and Remark 2.5 yield $\left|A(h) \nu_{\Sigma}\right|_{\Pi} \mid \geq \frac{1}{2}$ on $\overline{\Sigma_{a / 3}}$. Thus we can extend $\nu_{\Sigma}$ by $\left.\nu_{\Sigma}\right|_{\Pi}$ smoothly to $\overline{\Sigma_{a / 3}}$ and obtain the assertion by Lemma 2.6 .

\subsection{Mean Curvature}

For $h \in U$ we denote by $H_{\Gamma_{h}}$ the mean curvature of $\Gamma_{h}=\Theta_{h}(\Sigma)$ with respect to $\nu_{\Gamma_{h}}$. This subsection is devoted to the transformed mean curvature $K(h):=H_{\Gamma_{h}} \circ \Theta_{h}: \Sigma \rightarrow \mathbb{R}$. In particular we show that $K(h)$ has a quasilinear structure in terms of $h$ and the principal part for $h=0$ is given by the Laplace-Beltrami operator $\Delta_{\Sigma}$.

Lemma 2.8 Let $p>n+2$. Then there are $P \in C^{1}\left(U ; \mathcal{L}\left(X_{1}, X_{0}\right)\right)$ and $Q \in C^{1}\left(U ; X_{0}\right)$ with $P(0)=\Delta_{\Sigma}$ and $K(h)=P(h) h+Q(h)$ for all $h \in U \cap X_{1}$.

Proof. Let $\varphi_{l}: V_{l} \rightarrow U_{l} \subseteq \Sigma$ for $l=1, \ldots, N$ be suitable parametrizations of $\Sigma$ with $\Sigma=\bigcup_{l=1}^{N} U_{l}$ and $V_{l} \subseteq \mathbb{R}^{n-1}$. Then $\tilde{\varphi}_{l}:=\left(\varphi_{l}\right.$, id $): V_{l} \times(-a, a) \rightarrow U_{l} \times(-a, a)$ are appropriate parametrizations of $\Sigma \times(-a, a)$. Now fix $l$. The Euclidean metric $g^{\text {eucl }}$ on $\Sigma_{a}$ induces via the diffeomorphism $X$ from Subsection 2.2 a Riemannian metric $g^{X}$ on $\Sigma \times(-a, a)$. We denote by $w_{i j}, \Gamma_{i j}^{k}$ for $i, j, k=1, \ldots, n$ the local representation of $g^{X}$ and the Christoffel symbols with respect to $\tilde{\varphi}_{l}$, respectively, and $w^{i j}$ is as usual. Moreover, we set $\left.w_{i j}(h)\right|_{s}:=\left.w_{i j}\right|_{(s, h(s))}$ for $s \in U_{l}$. $\Gamma_{i j}^{k}(h)$ and $w^{i j}(h)$ are defined analogously. As in Escher and Simonett [8], proof of Lemma 3.1 on p. 274 ff. and Remark 3.2 on p. 277 it follows that

$$
\left.K(h)\right|_{U_{l}}=P_{l}(h) h+Q_{l}(h)
$$

where $P_{l}$ and $Q_{l}$ are altered by a factor $-(n-1)$ here. Additionally let $p_{j k}, p_{j}, q, l$. be as in Escher and Simonett [8] modified by the same factor. Then $P_{l}(0)$ is a local representation of $\Delta_{\Sigma}$. One can show that the $\left.w_{i j}\right|_{(s, r)}$ and $\left.\partial_{i} \partial_{j} X \cdot \partial_{m} X\right|_{(s, r)}$ for $i, j, m=$ $1, \ldots, n$ are at most quadratic in $r \in(-a, a)$ with smooth coefficients in $s \in \overline{U_{l}}$. Because of $\|h\|_{\infty}<a_{0}<\frac{a}{4}$ and $w_{i j}(h)=w_{i j}(., h()$.$) for i, j=1, \ldots, n$, a compactness argument yields

$$
\operatorname{det}\left(\left(w_{i j}(h)\right)_{i, j=1}^{n}\right) \geq c>0
$$

for a $c>0$ independent of $h$.

Using $X_{\gamma} \hookrightarrow C^{2}(\Sigma)$, the product rule for the Fréchet-derivative, the inverse formula as well as Lemma A.3 for the local representations in Escher and Simonett [8] implies

$$
p_{j k}(.) \circ \varphi_{l}, p_{i}(.) \circ \varphi_{l}, q(.) \circ \varphi_{l} \in C^{1}\left(U ; C^{1}\left(\overline{V_{l}}\right)\right) \quad \text { for } i, j, k=1, \ldots, n-1 .
$$

Now the claim follows utilizing a suitable partition of unity.

Furthermore, we need properties of $K$, when we insert a time-dependent height function $h \in V_{T}$. This gives us 


\section{Preliminaries}

Lemma 2.9 Let $p>n+2,1<q<p$ and $0<T \leq T_{0}$. Then it holds

$$
K \in B C^{1}\left(V_{T} ; W_{q}^{1-\frac{1}{q}, \frac{1}{2}\left(1-\frac{1}{q}\right)}\left(\Sigma_{T}\right)\right) .
$$

Moreover, $K$ and its derivative are bounded by a constant $C\left(p, q, T_{0}\right)>0$ when we add the term $\|h(0)\|_{X_{\gamma}}$ in the $\mathbb{E}_{1}(T)$-norm.

Proof. We use the same notation as in the proof of Lemma 2.8, Then

$$
\left.K(h)\right|_{U_{l}}=\sum_{\alpha \in \mathbb{N}_{0}^{n-1},|\alpha| \leq 2} a_{\alpha}\left(., h, \nabla_{s} h\right) \partial_{s}^{\alpha} h,
$$

where the $a_{\alpha}$ correspond to $p_{i j}, p_{j}$ and $q$, respectively, and $\partial_{s}^{\alpha}$ is the $\alpha$-th derivative with respect to the canonical coordinates.

First we estimate the $a_{\alpha}$ in suitable Hölder spaces. As in the proof of Lemma 2.6 one can show $\mathbb{E}_{1}(T) \hookrightarrow C^{\tau}\left([0, T] ; C^{2}(\Sigma)\right) \cap C^{\frac{1}{2}+\tau}\left([0, T] ; C^{1}(\Sigma)\right)$ for $\tau>0$ small because of $p>n+2$ and the embedding constant is bounded independent of $T>0$ if we add the term $\|h(0)\|_{X_{\gamma}}$ in the $\mathbb{E}_{1}(T)$-norm. Since the $w_{i j}$ for $i, j=1, \ldots, n$ are at most quadratic in $h$ with smooth coefficients in $\overline{U_{l}}$ product estimates in Hölder spaces yield

$$
w_{i j}(.) \circ \varphi_{l} \in B C^{1}\left(V_{T} ; C^{\tau}\left([0, T] ; C^{2}\left(\overline{V_{l}}\right)\right) \cap C^{\frac{1}{2}+\tau}\left([0, T] ; C^{1}\left(\overline{V_{l}}\right)\right)\right) \quad \text { for } i, j=1, \ldots, n .
$$

Now (2.17) implies 1 using the inverse formula, product estimates and Lemma A.3 that

$$
w^{i j}(.) \circ \varphi_{l} \in B C^{1}\left(V_{T} ; C^{\tau}\left([0, T] ; C^{1}\left(\overline{V_{l}}\right)\right) \cap C^{\frac{1}{2}+\tau}\left([0, T] ; C^{0}\left(\overline{V_{l}}\right)\right)\right) \quad \text { for } i, j=1, \ldots, n .
$$

Hence this also holds for $\Gamma_{i j}^{k}(.) \circ \varphi_{l}$ and $(l .)^{r}$, where $i, j, k=1, \ldots, n$ and $r \in \mathbb{R}$. Using the mapping properties in the local representations, we obtain for $\alpha \in \mathbb{N}_{0}^{n-1},|\alpha| \leq 2$

$$
a_{\alpha} \in B C^{1}\left(V_{T} ; C^{\tau}\left([0, T] ; C^{1}\left(\overline{V_{l}}\right)\right) \cap C^{\frac{1}{2}+\tau}\left([0, T] ; C^{0}\left(\overline{V_{l}}\right)\right)\right) .
$$

Furthermore, all above terms and their derivatives are bounded by a $C>0$ independent of $T>0$ if we add $\|h(0)\|_{X_{\gamma}}$ in the $\mathbb{E}_{1}(T)$-norm. So this also holds for the $a_{\alpha}$. The exponent $q$ does not appear here.

To show the desired properties of $K$ we use (2.18). Therefore we need another embedding for $\mathbb{E}_{1}(T)$ to estimate $\partial_{s}^{\alpha} h$ suitably. Theorem $\mathrm{A} .5$ and a well-known interpolation inequality for real interpolation spaces (see Corollary 1.7 in Lunardi [17]) yield

$$
\mathbb{E}_{1}(T) \hookrightarrow B_{p, \infty}^{1-\theta}\left(0, T ;\left(X_{0}, X_{1}\right)_{\theta, p}\right)
$$

for $\theta \in(0,1)$ and the embedding constant is bounded independent of $T>0$. We set $\theta=\frac{1}{2}\left(1+\frac{1}{p}\right)+\varepsilon$ for $\varepsilon>0$ small. Then for $\varepsilon=\varepsilon(p, q)>0$ small it holds that

$$
1-\frac{1}{p}+2 \theta=2+2 \varepsilon \quad \text { and } \quad 1-\theta=\frac{1}{2}\left(1-\frac{1}{p}\right)-\varepsilon>\frac{1}{2}\left(1-\frac{1}{q}\right) .
$$

\footnotetext{
${ }^{1}$ In fact, the same regularity as for the $w_{i j}(.) \circ \varphi_{l}$ should be obtained. But this is not needed.
} 
We infer $\mathbb{E}_{1}(T) \hookrightarrow B_{p, \infty}^{\frac{1}{2}\left(1-\frac{1}{p}\right)-\varepsilon}\left(0, T ; W_{p}^{2}(\Sigma)\right)$ and, again, the embedding constant is bounded independent of $T>0$. This implies

$$
\partial_{x_{i}}\left(. \circ \varphi_{l}\right), \partial_{x_{j}} \partial_{x_{i}}\left(. \circ \varphi_{l}\right) \in B C^{1}\left(V_{T} ; B_{p, \infty}^{\frac{1}{2}\left(1-\frac{1}{p}\right)-\varepsilon}\left(0, T ; L^{p}\left(V_{l}\right)\right) \cap L^{p}\left(0, T ; W_{p}^{1-\frac{1}{p}}\left(V_{l}\right)\right)\right)
$$

for $i, j=1, \ldots, n-1$ and the mappings and their derivatives are bounded independent of $T>0$. Since pointwise multiplication is a product on $C^{1}\left(\overline{V_{l}}\right) \times W_{p}^{1-\frac{1}{p}}\left(V_{l}\right) \rightarrow W_{p}^{1-\frac{1}{p}}\left(V_{l}\right)$ in sense of Definition A.1, using Lemma A.4 and (2.18)-(2.19) yields

$$
K(.) \circ \varphi_{l} \in B C^{1}\left(V_{T} ; B_{p, \infty}^{\frac{1}{2}\left(1-\frac{1}{p}\right)-\varepsilon}\left(0, T ; L^{p}\left(V_{l}\right)\right) \cap L^{p}\left(0, T ; W_{p}^{1-\frac{1}{p}}\left(V_{l}\right)\right)\right)
$$

where $K(.) \circ \varphi_{l}$ and the derivative are bounded by a constant $C>0$ independent of $T>0$. Now we use the embeddings $L^{p}\left(V_{l}\right) \hookrightarrow L^{q}\left(V_{l}\right)$ and $W_{p}^{1-\frac{1}{p}}\left(V_{l}\right) \hookrightarrow W_{q}^{1-\frac{1}{q}}\left(V_{l}\right)$ as well as

$$
B_{p, \infty}^{\frac{1}{2}\left(1-\frac{1}{p}\right)-\varepsilon}\left(0, T ; L^{p}\left(V_{l}\right)\right) \hookrightarrow B_{q, \infty}^{\frac{1}{2}\left(1-\frac{1}{p}\right)-\varepsilon}\left(0, T ; L^{p}\left(V_{l}\right)\right)
$$

where the latter can be shown by using Hölder's inequality and the embedding constant is bounded by $C\left(p, q, T_{0}\right)>0$. We infer from Simon [21, Corollary 15 that

$$
B_{q, \infty}^{\frac{1}{2}\left(1-\frac{1}{p}\right)-\varepsilon}\left(0, T ; L^{q}\left(V_{l}\right)\right) \hookrightarrow W_{q}^{\frac{1}{2}\left(1-\frac{1}{q}\right)}\left(0, T ; L^{q}\left(V_{l}\right)\right)
$$

and the embedding constant is also bounded by a $C\left(p, q, T_{0}\right)>0$. The claim follows by using a suitable partition of unity.

\section{Two-Phase Navier-Stokes System for Given Time-Dependent Interface}

We consider the Navier-Stokes part (2.6)-(2.11) for a fixed time-dependent height function $h \in V_{T}$ (or equivalently for a given family of hypersurfaces $\left\{\Gamma_{h}(t)\right\}_{t \in(0, T)}$ ) and show unique solvability and some properties of the solution operator. Let as before $p>n+2, R_{0}>0$ be fixed as well as $X_{0}, X_{1}, X_{\gamma}, U, \mathbb{E}_{1}(T)$ and $V_{T}$ for $T>0$ as in Subsection 2.3. Moreover, let $2<q<3$ and $h \in V_{T}$ with $h_{0}:=h(0)$. Then we set

$$
Y_{\gamma, h_{0}}:=\left\{v \in L^{p}(\Omega):\left.v\right|_{\tilde{\Theta}_{h_{0}}\left(\Omega^{ \pm}\right)} \in W_{q}^{2-\frac{2}{q}}\left(\tilde{\Theta}_{h_{0}}\left(\Omega^{ \pm}\right)\right)\right\}^{n} \cap W_{q, 0}^{1}(\Omega)^{n} \quad \text { and } \quad Y_{\gamma}:=Y_{\gamma, 0} .
$$

Now let $v_{0} \in Y_{\gamma, h_{0}} \cap L_{\sigma}^{q}(\Omega)$. In order to write the Navier-Stokes part (2.6)-(2.11) as an abstract fixed-point equation, we rearrange the equations such that the left hand side is the same as for the linear Stokes system in Appendix B. This yields

$$
\begin{aligned}
\partial_{t} u-\mu^{ \pm} \Delta u+\nabla \tilde{q} & =a^{ \pm}\left(h ; D_{x}\right)(u, \tilde{q})-u \cdot \nabla_{h} u+\nabla_{h} u \partial_{t} \tilde{\Theta}_{h} & & \text { in } \Omega^{ \pm} \times(0, T), \\
\operatorname{div} u & =\operatorname{Tr}\left(\nabla u-\nabla_{h} u\right)=: g(h) u & & \text { in } \Omega^{ \pm} \times(0, T), \\
\llbracket T(u, \tilde{q}) \rrbracket \nu_{\Sigma} & =t^{ \pm}\left(h ; D_{x}\right)(u, \tilde{q})-\sigma K(h) \nu_{h} & & \text { on } \Sigma \times(0, T), \\
\llbracket u \rrbracket & =0 & & \text { on } \Sigma \times(0, T), \\
\left.u\right|_{\partial \Omega} & =0 & & \text { on } \partial \Omega \times(0, T), \\
\left.u\right|_{t=0} & =v_{0} \circ \tilde{\Theta}_{h_{0}} & & \text { in } \Omega,
\end{aligned}
$$


where $A(h)=D \tilde{\Theta}_{h}^{-\top}, \nabla_{h}=A(h) \nabla$ and $K(h), \nu_{h}$ are as in Subsection 2.4 as well as

$$
\begin{aligned}
& a^{ \pm}\left(h ; D_{x}\right)(u, \tilde{q}):=\mu^{ \pm}\left(\operatorname{div}_{h} \nabla_{h} u-\Delta u\right)+\left(\nabla-\nabla_{h}\right) \tilde{q}, \\
& t^{ \pm}\left(h ; D_{x}\right)(u, \tilde{q}):=\llbracket 2 \mu^{ \pm} \operatorname{sym}(\nabla u)-\tilde{q} I \rrbracket\left(\nu_{\Sigma}-\nu_{h}\right)+\llbracket 2 \mu^{ \pm} \operatorname{sym}\left(\nabla u-\nabla_{h} u\right) \rrbracket \nu_{h} .
\end{aligned}
$$

We introduce the space $Y_{T, h_{0}, v_{0}}:=\left\{(u, \tilde{q}) \in Y_{T}:\left.u\right|_{t=0}=v_{0} \circ \tilde{\Theta}_{h_{0}}\right\}$ for the transformed velocity and pressure $(u, \tilde{q})$, where $Y_{T}:=Y_{T}^{1} \times Y_{T}^{2}$ and with $\Omega_{0}:=\Omega^{+} \cup \Omega^{-}$we define

$$
\begin{aligned}
& Y_{T}^{1}:=W_{q}^{1}\left(0, T ; L^{q}(\Omega)\right)^{n} \cap L^{q}\left(0, T ; W_{q}^{2}\left(\Omega_{0}\right) \cap W_{q, 0}^{1}(\Omega)\right)^{n}, \\
& Y_{T}^{2}:=\left\{\tilde{q} \in L^{q}\left(0, T ; W_{q,(0)}^{1}\left(\Omega_{0}\right)\right): \llbracket \tilde{q} \rrbracket \in W_{q}^{1-\frac{1}{q}, \frac{1}{2}\left(1-\frac{1}{q}\right)}\left(\Sigma_{T}\right)\right\} .
\end{aligned}
$$

Then the existence result for the Navier-Stokes part reads as follows:

Theorem 3.1 Let $p>n+2$ and $2<q<3$ with $1+\frac{n+2}{p}>\frac{n+2}{q}$. Then for $R>0$ there are $0<\varepsilon=\varepsilon(R)<R_{0}$ and $T_{0}=T_{0}(R)>0$ such that for all $0<T \leq T_{0}$ and

$$
h \in V_{T, \varepsilon}:=\left\{h \in V_{T}:\|h(0)\|_{X_{\gamma}} \leq \varepsilon\right\}, \quad v_{0} \in Y_{\gamma, h(0)} \cap L_{\sigma}^{q}(\Omega) \text { with }\left\|v_{0}\right\|_{Y_{\gamma, h(0)}} \leq R
$$

the Navier-Stokes part (3.1) -(3.6) has a unique solution $F_{T}\left(h, v_{0}\right):=(u, \tilde{q})(h) \in Y_{T, h(0), v_{0}}$ and it holds that $\left\|F_{T}\left(h, v_{0}\right)\right\|_{Y_{T}} \leq C_{R}$ as well as $\left.F_{T}\left(h, v_{0}\right)\right|_{[0, \tilde{T}]}=F_{\tilde{T}}\left(\left.h\right|_{[0, \tilde{T}]}, v_{0}\right)$ for all $0<\tilde{T} \leq T$.

Additionally, for $h_{j} \in V_{T, \varepsilon}$ with $h_{0}^{j}:=h_{j}(0)$ as well as $v_{0}^{j} \in Y_{\gamma, h_{j}(0)} \cap L_{\sigma}^{q}(\Omega)$ with $\left\|v_{0}^{j}\right\|_{Y_{\gamma, h_{j}(0)}} \leq R$ and $\tilde{v}_{0}^{j}:=v_{0}^{j} \circ \tilde{\Theta}_{h_{0}^{j}}$ for $j=1,2$ we have the estimate

$$
\left\|F_{T}\left(h_{1}, v_{0}^{1}\right)-F_{T}\left(h_{2}, v_{0}^{2}\right)\right\|_{Y_{T}} \leq \tilde{C}_{R}\left(\left\|h_{1}-h_{2}\right\|_{\mathbb{E}_{1}(T)}+\left\|h_{0}^{1}-h_{0}^{2}\right\|_{X_{\gamma}}+\left\|\tilde{v}_{0}^{1}-\tilde{v}_{0}^{2}\right\|_{Y_{\gamma}}\right) .
$$

For the proof we reformulate (3.1)-(3.6) as an abstract fixed-point equation

$$
w=L^{-1} G\left(w ; h, v_{0}\right) \quad \text { for } w \in Y_{T, h(0), v_{0}},
$$

where for $w=(u, \tilde{q}) \in Y_{T}$ we define

$$
L w:=\left(\begin{array}{c}
\partial_{t} u-\mu^{ \pm} \Delta u+\nabla \tilde{q} \\
\operatorname{div} u \\
\llbracket T(u, \tilde{q}) \rrbracket \nu_{\Sigma} \\
\left.u\right|_{t=0}
\end{array}\right), G\left(w ; h, v_{0}\right):=\left(\begin{array}{c}
a^{ \pm}\left(h ; D_{x}\right) w-u \cdot \nabla_{h} u+\nabla_{h} u \partial_{t} \tilde{\Theta}_{h} \\
g(h) u-\frac{1}{|\Omega|} \int_{\Omega} g(h) u d x \\
t^{ \pm}\left(h ; D_{x}\right) w-\sigma K(h) \nu_{h} \\
v_{0} \circ \tilde{\Theta}_{h(0)}
\end{array}\right) .
$$

Lemma B.1 and Theorem B.3 imply that $L: Y_{T} \rightarrow Z_{T}$ is an isomorphism, where

$$
Z_{T}:=\left\{\left(f, g, a, u_{0}\right) \in \tilde{Z}_{T}: \operatorname{div} u_{0}=\left.g\right|_{t=0} \text { in } W_{q,(0)}^{-1}(\Omega)\right\}
$$

with $\tilde{Z}_{T}:=\tilde{Z}_{T}^{1} \times \tilde{Z}_{T}^{2} \times\left(\tilde{Z}_{T}^{3}\right)^{n} \times Y_{\gamma}$ and the spaces

$$
\begin{aligned}
& \tilde{Z}_{T}^{1}:=L^{q}\left(0, T ; L^{q}(\Omega)\right)^{n}, \quad \tilde{Z}_{T}^{3}:=W_{q}^{1-\frac{1}{q}, \frac{1}{2}\left(1-\frac{1}{q}\right)}\left(\Sigma_{T}\right), \\
& \tilde{Z}_{T}^{2}:=\left\{g \in L^{q}\left(0, T ; W_{q,(0)}^{1}\left(\Omega_{0}\right)\right) \cap W_{q}^{1}\left(0, T ; W_{q,(0)}^{-1}(\Omega)\right): \operatorname{tr}_{\Sigma}\left(\left.g\right|_{\Omega^{+}}\right) \in \tilde{Z}_{T}^{3}\right\} .
\end{aligned}
$$

Here $Y_{T}, Z_{T}$ and $\tilde{Z}_{T}$ are Banach spaces with canonical norms, see Remark B.2, 
At this point we see why we have to apply 2 the fixed-point argument in the space $Y_{T, h_{0}, v_{0}}$ which depends on $h_{0}$ and $v_{0}$. Without the additional condition for $\left.u\right|_{t=0}$ in $Y_{T, h(0), v_{0}}$ the compatibility condition for $G\left(w ; h, v_{0}\right)$ in $Z_{T}$ would not be fulfilled in general. But it is essential that $G\left(. ; h, v_{0}\right)$ maps to $Z_{T}$ so that we can apply $L^{-1}$. For this reason we also subtracted the mean value in the second component of $G\left(w ; h, v_{0}\right)$. In fact, (3.1)-(3.6) and the formulation (3.8) are equivalent because of

Remark 3.2 Let $2<q<3, u \in W_{q, 0}^{1}(\Omega)^{n}$ and $h \in U_{0}$ with $U_{0}$ as in Remark 2.5. Then

$$
\operatorname{div} u=g(h) u-\frac{1}{|\Omega|} \int_{\Omega} g(h) u d x \quad \Leftrightarrow \quad \operatorname{div} u=g(h) u .
$$

Proof. The second equation is equivalent to $\operatorname{div}_{h} u=0$. For $u \in W_{q, 0}^{1}(\Omega)^{n}$ holds $\int_{\Omega} \operatorname{div} u d x=0$ and this shows the reverse direction. The other part follows as in A. and Wilke [2], p. 51.

The crucial step for showing Theorem 3.1 is to prove the following lemma which states the properties of the right hand side $G$.

Lemma 3.3 Let $p>n+2$ and $2<q<3$ with $1+\frac{n+2}{p}>\frac{n+2}{q}$ as well as $0<T \leq 1$. Then for all $w \in Y_{T}, h \in V_{T}$ and $v_{0} \in Y_{\gamma, h(0)} \cap L_{\sigma}^{q}(\Omega)$ we have that $G\left(w ; h, v_{0}\right)$ is contained in $\tilde{Z}_{T}$ and for $w \in Y_{T, h(0), v_{0}}$ it holds $G\left(w ; h, v_{0}\right) \in Z_{T}$. If $\left\|v_{0}\right\|_{Y_{\gamma, h(0)}}$ is bounded by a constant $R>0$, then $\left\|G\left(0 ; h, v_{0}\right)\right\|_{\tilde{Z}_{T}} \leq C_{R}$.

Moreover, let $R_{1}, R_{2}>0$. Then for $0<\varepsilon<R_{0}$ and $h_{j} \in V_{T, \varepsilon}$ with $h_{0}^{j}:=h_{j}(0)$, $v_{0}^{j} \in Y_{\gamma, h_{j}(0)} \cap L_{\sigma}^{q}(\Omega)$ and $\tilde{v}_{0}^{j}:=v_{0}^{j} \circ \tilde{\Theta}_{h_{j}(0)}$ as well as $w_{j}=\left(u_{j}, \tilde{q}_{j}\right) \in Y_{T}$ with $u_{0}^{j}:=u_{j}(0)$, $\left\|u_{0}^{j}\right\|_{Y_{\gamma}} \leq R_{1}$ and $\left\|w_{j}\right\|_{Y_{T}} \leq R_{2}$ for $j=1,2$ the following estimate holds:

$$
\begin{array}{r}
\left\|G\left(w_{1} ; h_{1}, v_{0}^{1}\right)-G\left(w_{2} ; h_{2}, v_{0}^{2}\right)\right\|_{\tilde{Z}_{T}} \leq C_{R_{1}, R_{2}}\left(T^{\delta}+\varepsilon\right)\left(\left\|w_{1}-w_{2}\right\|_{Y_{T}}+\left\|u_{0}^{1}-u_{0}^{2}\right\|_{Y_{\gamma}}\right)+ \\
+C_{R_{1}, R_{2}}\left(\left\|h_{1}-h_{2}\right\|_{\mathbb{E}_{1}(T)}+\left\|h_{0}^{1}-h_{0}^{2}\right\|_{X_{\gamma}}\right)+\left\|\tilde{v}_{0}^{1}-\tilde{v}_{0}^{2}\right\|_{Y_{\gamma}},
\end{array}
$$

where $\delta:=\min \left\{\frac{\tau}{2}, \frac{1}{q}-\frac{1}{p}\right\}>0$ and $\tau>0$ is as in Lemma [2.6.

Also as preparation for the proof of Lemma 3.3, in the following we discuss where the conditions for the exponent $q$ come from:

Remark 3.4 First of all, we have the restriction $2 \leq q<3$ because of Theorem B.3 below. Theorem 3.1 gives us the velocity field $u(h) \in Y_{T}^{1}$ in dependence of the height function $h$. Later we will insert it in equation (2.12) which should hold in $L^{p}\left(0, T ; X_{0}\right)$. In order to show local well-posedness afterwards, we need enough regularity for $\operatorname{tr}_{\Sigma} u$. This means

$$
\operatorname{tr}_{\Sigma} u \in L^{s}\left(0, T ; X_{0}\right)^{n} \quad \text { for some } s>p
$$

Since $Y_{\gamma}=\left\{v \in L^{p}(\Omega):\left.v\right|_{\Omega^{ \pm}} \in W_{q}^{2-\frac{2}{q}}\left(\Omega^{ \pm}\right)\right\}^{n} \cap W_{q, 0}^{1}(\Omega)^{n}$, we get from Lemma A.8 below that

$$
Y_{T}^{1} \hookrightarrow C^{0}\left([0, T] ; Y_{\gamma}\right) \cap L^{q}\left(0, T ; W_{q}^{2}\left(\Omega_{0}\right)^{n}\right)
$$

\footnotetext{
${ }^{2}$ A. and Wilke [2] transform with $\tilde{\Theta}_{h} \circ \tilde{\Theta}_{h_{0}}^{-1}$. Then for fixed $h_{0}$ one could omit the dependence on $v_{0}$. But here we have chosen the other strategy for the following reasons: On one hand it is difficult to obtain suitable properties of $\tilde{\Theta}_{h_{0}}^{-1}$ and on the other hand $\Gamma_{0}:=\tilde{\Theta}_{h_{0}}(\Sigma)$ would have the role of $\Sigma$ in Theorem B.3 but one needs a priori $C^{2,1}$-regularity for the interface there, see Shimizu [20].
} 
and the embedding constant is bounded independent of $T>0$ if we add the term $\|u(0)\|_{Y_{\gamma}}$ in the $Y_{T}^{1}$-norm. Now, for $\theta \in(0,1)$ we use the reiteration theorem in Lunardi [17], Corollary 1.24 to the result

$$
\left(W_{q}^{2-\frac{2}{q}}\left(\Omega^{ \pm}\right), W_{q}^{2}\left(\Omega^{ \pm}\right)\right)_{\theta, q}=\left(L^{q}\left(\Omega^{ \pm}\right), W_{q}^{2}\left(\Omega^{ \pm}\right)\right)_{(1-\theta)\left(1-\frac{1}{q}\right)+\theta, q}=B_{q, q}^{2-2(1-\theta) \frac{1}{q}}\left(\Omega^{ \pm}\right) .
$$

Therefore Lemma A.5yields $\left.Y_{T}^{1}\right|_{\Omega^{ \pm}} \hookrightarrow L^{s}\left(0, T ; B_{q, q}^{2-2(1-\theta) \frac{1}{q}}\left(\Omega^{ \pm}\right)^{n}\right)$ with $s=\frac{q}{\theta}$. To achieve (3.9) we want to choose $\theta \in(0,1)$ such that $\frac{q}{\theta}=s>p$ and that the Besov-space embeds into $W_{p}^{1}\left(\Omega^{ \pm}\right)^{n}$. Because of $q \leq p$ the latter is fulfilled for

$$
2-(1-\theta) \frac{2}{q}-\frac{n}{q}>1-\frac{n}{p} \quad \Leftrightarrow \quad \theta>-\frac{q}{2}-\frac{n q}{2 p}+\frac{n+2}{2} .
$$

Hence (3.9) holds by the trace theorem if

$$
\frac{q}{p}>-\frac{q}{2}-\frac{n q}{2 p}+\frac{n+2}{2} \Leftrightarrow \frac{q}{2}>-q \frac{n+2}{2 p}+\frac{n+2}{2} \Leftrightarrow 1>\frac{n+2}{q}-\frac{n+2}{p} .
$$

Since $p>n+2$ necessary conditions on $q$ are $q>2$, if $n=2$, and $q>\frac{5}{2}$, if $n=3$. So we directly wrote $q>2$ instead of $q \geq 2$. On the other hand the conditions are fulfilled for many $p, q$.

Another point is that we have to estimate $u \cdot \nabla_{h} u$ in the proof of Lemma 3.3 and therefore want to use the embedding $Y_{T}^{1} \hookrightarrow L^{r}\left(0, T ; C^{0}(\bar{\Omega})\right)^{n}$ for some $r>q$. Embeddings over $\Omega^{ \pm}$yield $Y_{\gamma} \hookrightarrow C^{0}(\bar{\Omega})^{n}$ for $q>2$, if $n=2$ and $q>\frac{5}{2}$, if $n=3$, since then $2-\frac{2+n}{q}>0$ holds. Hence from (3.10) we even obtain $Y_{T}^{1} \hookrightarrow C^{0}\left([0, T] ; C^{0}(\bar{\Omega})^{n}\right)$ and the embedding constant is bounded independent of $T>0$ if we add $\|u(0)\|_{Y_{\gamma}}$ in the $Y_{T}^{1}$-norm.

Altogether, for simplicity we restrict ourselves in Theorem 3.1 and Lemma 3.3 to exponents $p>n+2$ and $2<q<3$ with $1+\frac{n+2}{p}>\frac{n+2}{q}$.

Proof of Lemma 3.3. For simplicity we consider $0<T \leq 1$. Properties of $\tilde{\Theta}_{h}$ and $A(h)$ were shown in Lemma 2.6. The mean value theorem implies for all $h \in V_{T, \varepsilon}$ and $t \in[0, T]$

$$
\begin{aligned}
\left\|\left.A(h)\right|_{t}-I\right\|_{C^{1}(\bar{\Omega})^{n \times n}} & \leq\left\|\left.A(h)\right|_{t}-\left.A(h)\right|_{0}\right\|_{C^{1}(\bar{\Omega})^{n \times n}}+\left\|\left.A(h)\right|_{0}-I\right\|_{C^{1}(\bar{\Omega})^{n \times n}} \leq \\
& \leq C T^{\tau}+c\|h(0)\|_{X_{\gamma}} \leq C\left(T^{\tau}+\varepsilon\right) .
\end{aligned}
$$

Now let $w, h, v_{0}$ and $\left(u_{j}, \tilde{q}_{j}\right)=w_{j}, u_{0}^{j}$ as well as $h_{j}, h_{0}^{j}$ and $v_{0}^{j}, \tilde{v}_{0}^{j}$ for $j=1,2$ as in the lemma. We begin with the first component of $G$ which we have to estimate in $\tilde{Z}_{T}^{1}=L^{q}\left(0, T ; L^{q}(\Omega)^{n}\right)$. Lemma 2.6 implies $\left(\nabla-\nabla_{h}\right) \tilde{q} \in \tilde{Z}_{T}^{1}$ and (3.11) yields

$$
\begin{aligned}
& \left\|\left(\nabla-\nabla_{h_{1}}\right) \tilde{q}_{1}-\left(\left(\nabla-\nabla_{h_{2}}\right) \tilde{q}_{2}\right)\right\|_{\tilde{Z}_{T}^{1}} \leq\left\|\left(\nabla-\nabla_{h_{1}}\right)\left(\tilde{q}_{1}-\tilde{q}_{2}\right)+\left(\nabla_{h_{2}}-\nabla_{h_{1}}\right) \tilde{q}_{2}\right\|_{\tilde{Z}_{T}^{1}} \leq \\
& \leq C\left(T^{\tau}+\varepsilon\right)\left\|\tilde{q}_{1}-\tilde{q}_{2}\right\|_{Y_{T}^{2}}+C\left(\left\|h_{1}-h_{2}\right\|_{\mathbb{E}_{1}(T)}+\left\|h_{0}^{1}-h_{0}^{2}\right\|_{X_{\gamma}}\right) R_{2} .
\end{aligned}
$$

From Lemma 2.6 we obtain $\mu^{ \pm}\left(\operatorname{div}_{h} \nabla_{h} u-\Delta u\right) \in \tilde{Z}_{T}^{1}$ and null additions imply

$$
\begin{aligned}
& \left\|\mu^{ \pm}\left(\operatorname{div}_{h_{1}} \nabla_{h_{1}} u_{1}-\Delta u_{1}\right)-\mu^{ \pm}\left(\operatorname{div}_{h_{2}} \nabla_{h_{2}} u_{2}-\Delta u_{2}\right)\right\|_{\tilde{Z}_{T}^{1}} \leq \\
\leq & c\left(\left\|h_{1}-h_{2}\right\|_{\mathbb{E}_{1}(T)}+\left\|h_{0}^{1}-h_{0}^{2}\right\|_{X_{\gamma}}\right)\left\|u_{1}\right\|_{Y_{T}^{1}}+c\left\|A\left(h_{2}\right)-I\right\|_{C^{0}\left([0, T] ; C^{1}(\bar{\Omega})^{n \times n}\right)}\left\|u_{1}-u_{2}\right\|_{Y_{T}^{1}} .
\end{aligned}
$$


Together with this estimate we infer from (3.11)

$$
\begin{aligned}
& \left\|a^{ \pm}\left(h_{1}, D_{x}\right) w_{1}-a^{ \pm}\left(h_{2}, D_{x}\right) w_{2}\right\|_{\tilde{Z}_{T}^{1}} \leq \\
& \quad \leq C\left(T^{\tau}+\varepsilon\right)\left\|w_{1}-w_{2}\right\|_{Y_{T}}+C_{R_{2}}\left(\left\|h_{1}-h_{2}\right\|_{\mathbb{E}_{1}(T)}+\left\|h_{0}^{1}-h_{0}^{2}\right\|_{X_{\gamma}}\right) .
\end{aligned}
$$

Furthermore, Remark 3.4 yields $Y_{T}^{1} \hookrightarrow C^{0}\left([0, T] ; Y_{\gamma}\right) \hookrightarrow C^{0}\left([0, T] ; C^{0}(\bar{\Omega})\right)^{n}$ and the embedding constant is bounded independent of $T>0$ if we add $\|u(0)\|_{Y_{\gamma}}$ in the $Y_{T^{-}}^{1}$ norm. Hence we get $u \cdot \nabla_{h} v \in \tilde{Z}_{T}^{1}$ for $u, v \in Y_{T}^{1}$ because of Lemma 2.6] and null additions imply

$$
\begin{aligned}
& \left\|u_{1} \cdot \nabla_{h_{1}} u_{1}-u_{2} \cdot \nabla_{h_{2}} u_{2}\right\|_{\tilde{Z}_{T}^{1}} \leq \\
& \quad \leq C_{R_{1}, R_{2}} T^{\frac{1}{q}}\left(\left\|u_{1}-u_{2}\right\|_{Y_{T}^{1}}+\left\|u_{0}^{1}-u_{0}^{2}\right\|_{Y_{\gamma}}\right)+C_{R_{1}, R_{2}} T^{\frac{1}{q}}\left(\left\|h_{1}-h_{2}\right\|_{\mathbb{E}_{1}(T)}+\left\|h_{0}^{1}-h_{0}^{2}\right\|_{X_{\gamma}}\right) .
\end{aligned}
$$

Additionally we obtain $\nabla_{h} u \partial_{t} \tilde{\Theta}_{h} \in \tilde{Z}_{T}^{1}$ from Lemma 2.6, the embedding for $Y_{T}^{1}$ mentioned above and $W_{p}^{1}(\Omega) \hookrightarrow C^{0}(\bar{\Omega})$ as $p>n+2$. Moreover, null additions yield

$$
\begin{aligned}
& \left\|\nabla_{h_{1}} u_{1} \partial_{t} \tilde{\Theta}_{h_{1}}-\nabla_{h_{2}} u_{2} \partial_{t} \tilde{\Theta}_{h_{2}}\right\|_{\tilde{Z}_{T}^{1}} \leq \\
& \quad \leq C_{R_{1}, R_{2}} T^{\frac{1}{q}-\frac{1}{p}}\left(\left\|h_{1}-h_{2}\right\|_{\mathbb{E}_{1}(T)}+\left\|h_{0}^{1}-h_{0}^{2}\right\|_{X_{\gamma}}\right)+c T^{\frac{1}{q}-\frac{1}{p}}\left(\left\|u_{1}-u_{2}\right\|_{Y_{T}^{1}}+\left\|u_{0}^{1}-u_{0}^{2}\right\|_{Y_{\gamma}}\right) .
\end{aligned}
$$

Now we consider the second component of $G$. First of all, Lemma 2.6 implies that $g(h) u=\operatorname{Tr}\left(\nabla u-\nabla_{h} u\right)=(I-A(h)): \nabla u \in L^{q}\left(0, T ; W_{q}^{1}\left(\Omega_{0}\right)\right)$ and (3.11) yields with a null addition

$\left\|g\left(h_{1}\right) u_{1}-g\left(h_{2}\right) u_{2}\right\|_{L^{q}\left(0, T ; W_{q}^{1}\left(\Omega_{0}\right)\right)} \leq c\left(T^{\tau}+\varepsilon\right)\left\|u_{1}-u_{2}\right\|_{Y_{T}^{1}}+c_{R_{2}}\left(\left\|h_{1}-h_{2}\right\|_{\mathbb{E}_{1}(T)}+\left\|h_{0}^{1}-h_{0}^{2}\right\|_{X_{\gamma}}\right)$.

Therefore this also follows for $G\left(w ; h, v_{0}\right)_{2}$ in $L^{q}\left(0, T ; W_{q,(0)}^{1}\left(\Omega_{0}\right)\right)$. Apart from that we have $G\left(w ; h, v_{0}\right)_{2}=g(h) u$ in $L^{q}\left(0, T ; W_{q,(0)}^{-1}(\Omega)\right)$ since constants drop out when integrating with $\phi \in W_{q^{\prime},(0)}^{1}(\Omega)$. In this space we need to show the existence of a weak derivative. To this end let $\eta \in C_{0}^{\infty}(0, T)$. We approximate $u$ and $A(h)$ suitably. Using convolution one can show that there are $\left(u^{m}\right)_{m \in \mathbb{N}}$ in $C^{\infty}\left([0, T] ; W_{q}^{2}\left(\Omega_{0}\right) \cap W_{q, 0}^{1}(\Omega)\right)^{n}$ and $\left(A^{m}\right)_{m \in \mathbb{N}}$ in $C^{\infty}\left([0, T] ; C^{1}(\bar{\Omega})^{n \times n}\right)$ with $u^{m} \rightarrow u$ in $Y_{T}^{1}$ and $A^{m} \rightarrow A(h)$ in the space $C^{0}\left([0, T] ; C^{1}(\bar{\Omega})^{n \times n}\right) \cap W_{p}^{1}\left(0, T ; L^{p}(\Omega)^{n \times n}\right)$ for $m \rightarrow \infty$. Now the product rule yields

$$
C^{\infty}\left([0, T] ; W_{q}^{1}\left(\Omega_{0}\right)\right) \ni g^{m}:=\left(I-A^{m}\right): \nabla u^{m} \stackrel{m \rightarrow \infty}{\longrightarrow} g(h) u \quad \text { in } L^{q}\left(0, T ; W_{q}^{1}\left(\Omega_{0}\right)\right) .
$$

Choose $\phi \in W_{q^{\prime},(0)}^{1}(\Omega)$ arbitrary. Then for $m \in \mathbb{N}$ we obtain

$$
-\int_{0}^{T} \partial_{t} \eta(t) \int_{\Omega} g^{m}(t) \phi d x d t=\int_{0}^{T} \eta(t) \int_{\Omega} \partial_{t} g^{m}(t) \phi d x d t .
$$

Here $\partial_{t} g^{m}=-\partial_{t} A^{m}: \nabla u^{m}+\left(I-A^{m}\right): \nabla \partial_{t} u^{m}$ and therefore

$$
\int_{\Omega} \partial_{t} g^{m} \phi d x=\int_{\Omega}-\partial_{t} A^{m}: \nabla u^{m} \phi+\partial_{t} u^{m} \cdot \operatorname{div} A^{m} \phi-\left(\left(I-A^{m}\right) \partial_{t} u^{m}\right) \cdot \nabla \phi d x,
$$


where we used integration by parts for the second term. In this equation we want to pass to the limit. Lemma A.8 implies $Y_{T}^{1} \hookrightarrow C^{0}\left([0, T] ; Y_{\gamma}\right)$ and the embedding constant is bounded independent of $T>0$ if we add $\|u(0)\|_{Y_{\gamma}}$ in the $Y_{T}^{1}$-norm. This yields

$$
\partial_{t} A^{m}: \nabla u^{m} \stackrel{m \rightarrow \infty}{\longrightarrow} \partial_{t} A(h): \nabla u \quad \text { in } L^{p}\left(0, T ; L^{r_{1}}(\Omega)\right) \quad \text { with } \frac{1}{r_{1}}:=\frac{1}{p}+\frac{1}{q} \in(0,1) .
$$

Furthermore we have $\partial_{t} u^{m} \cdot \operatorname{div} A^{m} \stackrel{m \rightarrow \infty}{\longrightarrow} \partial_{t} u \cdot \operatorname{div} A$ and $\left(I-A^{m}\right) \partial_{t} u^{m} \stackrel{m \rightarrow \infty}{\longrightarrow}(I-A(h)) \partial_{t} u$ in $L^{q}\left(0, T ; L^{q}(\Omega)\right)$ (vector-valued for the second one). Now it holds $W_{q^{\prime}}^{1}(\Omega) \hookrightarrow L^{r_{2}}(\Omega)$ for $n=2,3$ with $\frac{1}{r_{2}}:=\frac{1}{q^{\prime}}-\frac{1}{n} \in(0,1)$ and because of

$$
\frac{1}{r_{1}}+\frac{1}{r_{2}}=1+\frac{1}{p}-\frac{1}{n} \in(0,1) \quad \text { for } p>n+2, n=2,3
$$

the mapping $L^{r_{1}}(\Omega) \rightarrow W_{q,(0)}^{-1}(\Omega): g \mapsto\langle g,$.$\rangle is bounded and linear. This is also true for$

$$
L^{q}(\Omega) \rightarrow W_{q,(0)}^{-1}(\Omega): g \mapsto\langle g, .\rangle \quad \text { and } \quad L^{q}(\Omega)^{n} \rightarrow W_{q,(0)}^{-1}(\Omega): g \mapsto\left[\phi \mapsto \int_{\Omega} g \cdot \nabla \phi d x\right]
$$

Since $\langle., \phi\rangle: W_{q,(0)}^{-1}(\Omega) \rightarrow \mathbb{R}$ is bounded and linear for $\phi \in W_{q^{\prime},(0)}^{1}(\Omega)$ we can pass to the limit in (3.12) -(3.13) for fixed $\phi$ and then we put outside the duality product. Hence $g(h) u \in W_{q}^{1}\left(0, T ; W_{q,(0)}^{-1}(\Omega)\right)$ and the derivative is given by

$$
\left\langle\partial_{t}(g(h) u), \phi\right\rangle=\int_{\Omega}\left(-\partial_{t} A(h): \nabla u+\partial_{t} u \cdot \operatorname{div} A(h)\right) \phi-\left((I-A(h)) \partial_{t} u\right) \cdot \nabla \phi d x .
$$

For the difference of the derivatives the above observations together with null additions and $\operatorname{div} I=0$ yield

$$
\begin{aligned}
& \left\|\partial_{t}\left(g\left(h_{1}\right) u_{1}\right)-\partial_{t}\left(g\left(h_{2}\right) u_{2}\right)\right\|_{L^{q}\left(0, T ; W_{q,(0)}^{-1}(\Omega)\right)} \leq \\
& \quad \leq C T^{\frac{1}{q}-\frac{1}{p}}\left(C_{R_{1}, R_{2}}\left(\left\|h_{1}-h_{2}\right\|_{\mathbb{E}_{1}(T)}+\left\|h_{0}^{1}-h_{0}^{2}\right\|_{X_{\gamma}}\right)+\left\|u_{1}-u_{2}\right\|_{Y_{T}^{1}}+\left\|u_{0}^{1}-u_{0}^{2}\right\|_{Y_{\gamma}}\right)+ \\
& \quad+C_{R_{2}}\left(\left\|h_{1}-h_{2}\right\|_{\mathbb{E}_{1}(T)}+\left\|h_{0}^{1}-h_{0}^{2}\right\|_{X_{\gamma}}\right)+C\left(T^{\tau}+\varepsilon\right)\left\|u_{1}-u_{2}\right\|_{Y_{T}^{1}} .
\end{aligned}
$$

Now we show $\operatorname{tr}_{\Sigma}\left(\left.G\left(w ; h, v_{0}\right)_{2}\right|_{\Omega^{+}}\right) \in \tilde{Z}_{T}^{3}$ and the related difference estimate. To this end let $\operatorname{ext}_{T} \in \mathcal{L}\left(W_{q}^{2,1}\left(\Omega_{T}^{+}\right), W_{q}^{2,1}\left(\Omega^{+} \times \mathbb{R}_{+}\right)\right)$for $T>0$ be extension operators in time such that

$$
\left\|\operatorname{ext}_{T}(u)\right\|_{W_{q}^{2,1}\left(\Omega^{+} \times \mathbb{R}_{+}\right)} \leq M\left(\|u\|_{W_{q}^{2,1}\left(\Omega_{T}^{+}\right)}+\|u(0)\|_{W_{q}^{2-\frac{2}{q}}\left(\Omega^{+}\right)}\right)
$$

holds for some $M>0$. To construct such operators one can e.g. first extend suitably from $\Omega$ to $\mathbb{R}^{n}$ and then proceed as in Amann [4, Lemma 7.2 utilizing that the Laplaceoperator has maximal $L^{p}$-regularity, cf. Prüss and Simonett [18], Theorem 6.1.8.

We can extend $\operatorname{ext}_{T} u$ by reflection to a $\tilde{u}$ defined on $\Omega^{+} \times \mathbb{R}$. From Grubb [10], (A.8)(A.12) we know that $\nabla \tilde{u} \in H_{q}^{1, \frac{1}{2}}\left(\Omega^{+} \times \mathbb{R}\right)^{n \times n} \hookrightarrow W_{q}^{1, \frac{1}{2}}\left(\Omega^{+} \times \mathbb{R}\right)^{n \times n}$, where the latter embedding holds because of $q \in(2,3)$. Moreover, we have the estimate

$$
\|\nabla \tilde{u}\|_{W_{q}^{1, \frac{1}{2}}\left(\Omega^{+} \times \mathbb{R}\right)^{n \times n}} \leq C\|\tilde{u}\|_{W_{q}^{2,1}\left(\Omega^{+} \times \mathbb{R}\right)^{n}} \leq \tilde{C}\left(\|u\|_{Y_{T}^{1}}+\|u(0)\|_{Y_{\gamma}}\right) .
$$


We decompose $A(h)=\tilde{A}(h)+A(h(0))$ with $\tilde{A}(h):=A(h)-A(h(0))$ and extend $\tilde{A}(h)$ by reflection at $T$ to $[0,2 T]$ and then by 0 to all of $\mathbb{R}_{+}$. Then $\tilde{A}(h)$ is contained in $C^{\frac{1}{2}+\tau}\left(\mathbb{R}_{+} ; C^{0}(\bar{\Omega})^{n \times n}\right) \cap C^{\tau}\left(\mathbb{R}_{+} ; C^{1}(\bar{\Omega})^{n \times n}\right)$ and the norm is estimated by the respective one of $A(h)-A(h(0))$. Since $\tilde{A}(h)=0$ on $(2 T, \infty)$, we can apply Lemma A.4 to $I=(0,2 T+1)$ and $\left.\tilde{A}(h)\right|_{\Omega^{+}}$. Afterwards we extend by 0 and obtain

$$
\left.(I-A(h(0))-\tilde{A}(h))\right|_{\Omega^{+}}: \nabla \tilde{u} \in W_{q}^{1, \frac{1}{2}}\left(\Omega^{+} \times \mathbb{R}_{+}\right) .
$$

This then also holds for the mean value and e.g. Denk, Hieber and Prüss [6], Lemma 3.5 implies $\operatorname{tr}_{\Sigma}\left(\left.G\left(w ; h, v_{0}\right)_{2}\right|_{\Omega^{+}}\right) \in \tilde{Z}_{T}^{3}$. Furthermore, a null addition yields

$$
\begin{aligned}
& \left\|\operatorname{tr}_{\Sigma}\left(\left.G\left(w_{1} ; h_{1}, v_{0}^{1}\right)_{2}\right|_{\Omega^{+}}\right)-\operatorname{tr}_{\Sigma}\left(\left.G\left(w_{2} ; h_{2}, v_{0}^{2}\right)_{2}\right|_{\Omega^{+}}\right)\right\|_{\tilde{Z}_{T}^{3}} \leq \\
& \leq C\left(\left\|I-A\left(h_{0}^{1}\right)\right\|_{C^{1}(\bar{\Omega})^{n \times n}}+\left\|\tilde{A}\left(h_{1}\right)\right\|_{C^{\frac{1+\tau}{2}}\left(\mathbb{R}_{+} ; C^{0}(\bar{\Omega})\right) \cap C^{0}\left(\mathbb{R}_{+} ; C^{1}(\bar{\Omega})\right)^{n \times n}}\right) . \\
& \cdot\left\|\tilde{u}_{1}-\tilde{u}_{2}\right\|_{W_{q}^{2,1}\left(\Omega^{+} \times \mathbb{R}_{+}\right)^{n}}+\left\|A\left(h_{1}\right)-A\left(h_{2}\right)\right\|_{C^{\frac{1}{2}+\tau}\left([0, T] ; C^{0}(\bar{\Omega})\right) \cap C^{0}\left([0, T] ; C^{1}(\bar{\Omega})\right)^{n \times n}} C_{R_{1}, R_{2}} \leq \\
& \leq C\left(T^{\tau / 2}+\varepsilon\right)\left(\left\|u_{1}-u_{2}\right\|_{Y_{T}^{1}}+\left\|u_{0}^{1}-u_{0}^{2}\right\|_{Y_{\gamma}}\right)+C_{R_{1}, R_{2}}\left(\left\|h_{1}-h_{2}\right\|_{\mathbb{E}_{1}(T)}+\left\|h_{0}^{1}-h_{0}^{2}\right\|_{X_{\gamma}}\right),
\end{aligned}
$$

where we used Lemma 2.6, (3.11) and $T \leq 1$.

Next we study the third component of $G$. Properties of $\nu$. were shown in Corollary 2.7. As in (3.11) we obtain $\left\|\nu_{\Sigma}-\nu_{h}\right\|_{C^{0}\left([0, T] ; C^{1}(\Sigma)^{n}\right)} \leq C\left(T^{\tau}+\varepsilon\right)$. Similar as before we get $\llbracket 2 \mu^{ \pm} \operatorname{sym}(\nabla u) \rrbracket, \llbracket 2 \mu^{ \pm} \operatorname{sym}\left(\nabla u-\nabla_{h} u\right) \rrbracket \in\left(\tilde{Z}_{T}^{3}\right)^{n \times n}$ and the following estimates hold:

$$
\begin{aligned}
\left\|\llbracket 2 \mu^{ \pm} \operatorname{sym}(\nabla u) \rrbracket\right\|_{\left(\tilde{Z}_{T}^{3}\right)^{n \times n}} & \leq C\left(\|u\|_{Y_{T}^{1}}+\|u(0)\|_{Y_{\gamma}}\right), \\
\left\|\llbracket 2 \mu^{ \pm} \operatorname{sym}\left(\nabla u-\nabla_{h_{1}} u\right) \rrbracket\right\|_{\left(\tilde{Z}_{T}^{3}\right)^{n \times n}} & \leq C\left(T^{\tau / 2}+\varepsilon\right)\left(\|u\|_{Y_{T}^{1}}+\|u(0)\|_{Y_{\gamma}}\right), \\
\left\|\llbracket 2 \mu^{ \pm} \operatorname{sym}\left(\nabla_{h_{1}} u-\nabla_{h_{2}} u\right) \rrbracket\right\|_{\left(\tilde{Z}_{T}^{3}\right)^{n \times n}} & \leq C_{R_{1}, R_{2}}\left(\left\|h_{1}-h_{2}\right\|_{\mathbb{E}_{1}(T)}+\left\|h_{0}^{1}-h_{0}^{2}\right\|_{X_{\gamma}}\right) .
\end{aligned}
$$

Since by definition $\llbracket \tilde{q} \rrbracket \in \tilde{Z}_{T}^{3}$ and a product on $C^{1}(\Sigma) \times W_{q}^{1-\frac{1}{q}}(\Sigma) \rightarrow W_{q}^{1-\frac{1}{q}}(\Sigma)$ is given by pointwise multiplication, Lemma A.4 implies

$$
t^{ \pm}\left(h ; D_{x}\right) w=\llbracket 2 \mu^{ \pm} \operatorname{sym}(\nabla u)-\tilde{q} I \rrbracket\left(\nu_{\Sigma}-\nu_{h}\right)+\llbracket 2 \mu^{ \pm} \operatorname{sym}\left(\nabla u-\nabla_{h} u\right) \rrbracket \nu_{h} \in\left(\tilde{Z}_{T}^{3}\right)^{n} .
$$

Corollary 2.7 yields $\left[\nu_{h}\right]_{C^{\frac{1+\tau}{2}}\left([0, T] ; C^{0}(\Sigma)\right)^{n}} \leq C T^{\tau / 2}$ and with null additions we infer

$$
\begin{aligned}
& \left\|t^{ \pm}\left(h_{1} ; D_{x}\right) w_{1}-t^{ \pm}\left(h_{2} ; D_{x}\right) w_{2}\right\|_{\left(\tilde{Z}_{T}^{3}\right)^{n}} \leq \\
& \quad \leq C\left(T^{\tau / 2}+\varepsilon\right)\left(\left\|w_{1}-w_{2}\right\|_{Y_{T}}+\left\|u_{0}^{1}-u_{0}^{2}\right\|_{Y_{\gamma}}\right)+C_{R_{1}, R_{2}}\left(\left\|h_{1}-h_{2}\right\|_{\mathbb{E}_{1}(T)}+\left\|h_{0}^{1}-h_{0}^{2}\right\|_{X_{\gamma}}\right) .
\end{aligned}
$$

Invoking Lemma 2.9 and Corollary 2.7 together with Lemma A.4 and the product rule, we obtain $\sigma K(.) \nu$. $\in B C^{1}\left(V_{T} ;\left(\tilde{Z}_{T}^{3}\right)^{n}\right)$ where $\sigma K(.) \nu$. and the derivative is bounded independent of $0<T \leq 1$ if we add $\|h(0)\|_{X_{\gamma}}$ in the $\mathbb{E}_{1}(T)$-norm. In particular we get

$$
\left\|\sigma K\left(h_{1}\right) \nu_{h_{1}}-\sigma K\left(h_{2}\right) \nu_{h_{2}}\right\|_{\left(\tilde{Z}_{T}^{3}\right)^{n}} \leq C\left(\left\|h_{1}-h_{2}\right\|_{\mathbb{E}_{1}(T)}+\left\|h_{0}^{1}-h_{0}^{2}\right\|_{X_{\gamma}}\right) .
$$

For the fourth component we use that $\tilde{\Theta}_{h(0)}: \Omega \rightarrow \Omega$ is a $C^{2}$-diffeomorphism and the identity in a neighbourhood of $\partial \Omega$ by Lemma 2.4 and Remark 2.5. Moreover, because of Lemma 2.6 we have that $|\operatorname{det} A(h(0))|$ and the $C^{2}(\bar{\Omega})$-norm of $\tilde{\Theta}_{h(0)}$ are bounded by a 
constant independent of $h \in V_{T}$. Therefore $W_{q}^{k}\left(\tilde{\Theta}_{h(0)}\left(\Omega^{ \pm}\right)\right)^{n} \rightarrow W_{q}^{k}\left(\Omega^{ \pm}\right)^{n}: v \mapsto v \circ \tilde{\Theta}_{h(0)}$ for $k=0,1,2$ is bounded, linear and the operator norm is bounded independent of $h \in V_{T}$. The latter follows from Adams and Fournier [3], Theorem 3.41 and its proof. By interpolation this also holds for $Y_{\gamma, h(0)} \rightarrow Y_{\gamma}: v \mapsto v \circ \tilde{\Theta}_{h(0)}$. We obtain $G\left(w ; h, v_{0}\right)_{4}=$ $v_{0} \circ \tilde{\Theta}_{h(0)} \in Y_{\gamma}$ and, if $\left\|v_{0}\right\|_{Y_{\gamma, h(0)}} \leq R$ for some $R>0$, we have $\left\|v_{0} \circ \tilde{\Theta}_{h(0)}\right\|_{Y_{\gamma}} \leq C_{R}$. The estimate for the difference of $\tilde{v}_{0}^{j}$ is trivial.

Altogether we have proven $G\left(w ; h, v_{0}\right) \in \tilde{Z}_{T}$ and the difference estimate in the lemma. Additionally $G\left(0 ; h, v_{0}\right)=\left(0,0,-\sigma K(h) \nu_{h}, v_{0} \circ \tilde{\Theta}_{h(0)}\right)^{\top}$ and the above implies $\left\|G\left(0 ; h, v_{0}\right)\right\|_{\tilde{Z}_{T}} \leq$ $C_{R}$. It remains to show for $w \in Y_{T, h(0), v_{0}}$ the compatibility condition for $G\left(w ; h, v_{0}\right)$ in $Z_{T}$ which is equivalent to $\operatorname{div}(u(0))=\left.(g(h) u)\right|_{t=0}$ in $W_{q,(0)}^{-1}(\Omega)$ since constants drop out when integrating with $\phi \in W_{q^{\prime},(0)}^{1}(\Omega)$. Lemma A.8 implies that $Y_{T}^{1} \hookrightarrow C^{0}\left([0, T] ; Y_{\gamma}\right)$ and therefore

$$
g(h) u=(I-A(h)): \nabla u \in C^{0}\left([0, T] ; L^{q}(\Omega)\right) .
$$

Since $L^{q}(\Omega) \rightarrow W_{q,(0)}^{-1}(\Omega): g \mapsto\langle g,$.$\rangle is bounded and linear, it suffices to show \operatorname{div} u(0)=$ $g(h(0)) u(0)$ or, equivalently, $\operatorname{div}_{h(0)} u(0)=0$ in $L^{q}(\Omega)$. But by chain rule we know $\left(\operatorname{div} v_{0}\right) \circ \tilde{\Theta}_{h(0)}=\operatorname{div}_{h(0)}(u(0))$. Since $v_{0}$ is divergence-free, the claim follows.

After this preparation we can show the existence result for the Navier-Stokes part. Proof of Theorem 3.1. Let $p, q$ be as in the theorem and $R>0$ be arbitrary. Then for $h \in V_{T}$ and $v_{0} \in Y_{\gamma, h(0)} \cap L_{\sigma}^{q}(\Omega)$ with $\left\|v_{0}\right\|_{Y_{\gamma, h(0)}} \leq R$ Lemma 3.3 yields

$$
\left\|v_{0} \circ \tilde{\Theta}_{h(0)}\right\|_{Y_{\gamma}} \leq\left\|G\left(0 ; h, v_{0}\right)\right\|_{\tilde{Z}_{T}} \leq C_{R}
$$

We set $C_{L}:=\sup _{0<T \leq 1}\left\|L^{-1}\right\|_{\mathcal{L}\left(Z_{T}, Y_{T}\right)}$ and choose $R_{1}:=C_{R}$ and $R_{2}:=2 C_{R} C_{L}$ in Lemma 3.3. Here $C_{L}$ is well-defined because of Theorem B.3. Then (3.16) implies for $0<\varepsilon<R_{0}$ and $0<T \leq 1$ as well as $h \in V_{T, \varepsilon}$ and $w \in Y_{T, h(0), v_{0}}$ with $\|w\|_{Y_{T}} \leq R_{2}$ the estimate

$$
\begin{aligned}
\left\|L^{-1} G\left(w ; h, v_{0}\right)\right\|_{Y_{T}} & \leq\left\|L^{-1}\right\|_{\mathcal{L}\left(Z_{T}, Y_{T}\right)}\left(\left\|G\left(0 ; h, v_{0}\right)\right\|_{\tilde{Z}_{T}}+\left\|G\left(w ; h, v_{0}\right)-G\left(0 ; h, v_{0}\right)\right\|_{\tilde{Z}_{T}}\right) \leq \\
& \leq C_{L}\left(C_{R}+C_{R_{1}, R_{2}}\left(T^{\delta}+\varepsilon\right)\left(\|w\|_{Y_{T}}+\left\|v_{0} \circ \tilde{\Theta}_{h(0)}\right\|_{Y_{\gamma}}\right)\right)
\end{aligned}
$$

where $\delta>0$. For $T_{0}=T_{0}(R), \varepsilon=\varepsilon(R)>0$ small we obtain from (3.16) for all $0<T \leq T_{0}$

$$
\left\|L^{-1} G\left(w ; h, v_{0}\right)\right\|_{Y_{T}} \leq \frac{1}{2} R_{2}+\tilde{C}_{R}\left(T^{\delta}+\varepsilon\right) \leq \frac{2}{3} R_{2} .
$$

Lemma 3.3 yields $\left\|L^{-1} G\left(w_{1} ; h, v_{0}\right)-L^{-1} G\left(w_{2} ; h, v_{0}\right)\right\|_{Y_{T}} \leq C_{L} C_{R_{1}, R_{2}}\left(T_{0}^{\delta}+\varepsilon\right)\left\|w_{1}-w_{2}\right\|_{Y_{T}}$ for all $w_{j} \in Y_{T, h(0), v_{0}}$ with $\left\|w_{j}\right\|_{Y_{T}} \leq R_{2}$ for $j=1,2$. We choose $\varepsilon=\varepsilon(R)>0$ and $T_{0}=T_{0}(R)>0$ small such that

$$
C_{L} C_{R_{1}, R_{2}}\left(T_{0}^{\delta}+\varepsilon\right) \leq \frac{1}{2}
$$

holds. Then $L^{-1} G\left(. ; h, v_{0}\right): \overline{B_{Y_{T, h(0), v_{0}}}\left(0, R_{2}\right)} \rightarrow \overline{B_{Y_{T, h(0), v_{0}}}\left(0, R_{2}\right)}$ is well-defined for all $0<T \leq T_{0}$ and a strict contraction. Here $Y_{T, h(0), v_{0}}$ is a complete metric space as closed subset of a Banach space. Banach's fixed-point theorem yields a unique fixed-point $F_{T}\left(h, v_{0}\right)$ in $\overline{B_{Y_{T, h(0), v_{0}}}\left(0, R_{2}\right)}$ and it holds

$$
\left.F_{T}\left(h, v_{0}\right)\right|_{[0, \tilde{T}]}=F_{\tilde{T}}\left(\left.h\right|_{[0, \tilde{T}]}, v_{0}\right) \quad \text { for all } 0<\tilde{T} \leq T
$$


since $\left.h\right|_{[0, \tilde{T}]} \in V_{\tilde{T}, \varepsilon}$ as well as $\left.F_{T}\left(h, v_{0}\right)\right|_{[0, \tilde{T}]} \in \overline{B_{Y_{\tilde{T}, h(0), v_{0}}}\left(0, R_{2}\right)}$ and $L^{-1} G\left(. ; h, v_{0}\right)$ is compatible with restrictions in time on $[0, \tilde{T}]$ for $0<\tilde{T} \leq T$. The uniqueness in $Y_{T, h(0), v_{0}}$ can be shown with the compatibility for restrictions in time and (3.17).

It remains to prove the Lipschitz dependence. To this end let $h_{j}, h_{0}^{j}, v_{0}^{j}$ and $\tilde{v}_{0}^{j}$ for $j=1,2$ be as in the theorem. Then Lemma 3.3 implies

$$
\begin{aligned}
& \left\|F_{T}\left(h_{1}, v_{0}^{1}\right)-F_{T}\left(h_{2}, v_{0}^{2}\right)\right\|_{Y_{T}}=\left\|L^{-1}\left(G\left(F_{T}\left(h_{1}, v_{0}^{1}\right) ; h_{1}, v_{0}^{1}\right)-G\left(F_{T}\left(h_{2}, v_{0}^{2}\right) ; h_{2}, v_{0}^{2}\right)\right)\right\|_{Y_{T}} \leq \\
& \quad \leq\left\|L^{-1}\right\|_{\mathcal{L}\left(Z_{T}, Y_{T}\right)}\left\|G\left(F_{T}\left(h_{1}, v_{0}^{1}\right) ; h_{1}, v_{0}^{1}\right)-G\left(F_{T}\left(h_{2}, v_{0}^{2}\right) ; h_{2}, v_{0}^{2}\right)\right\|_{Z_{T}} \leq \\
& \quad \leq C_{L} C_{R_{1}, R_{2}}\left(T^{\delta}+\varepsilon\right)\left(\left\|F_{T}\left(h_{1}, v_{0}^{1}\right)-F_{T}\left(h_{2}, v_{0}^{2}\right)\right\|_{Y_{T}}+\left\|\tilde{v}_{0}^{1}-\tilde{v}_{0}^{2}\right\|_{Y_{\gamma}}\right)+ \\
& \quad+C_{L} C_{R_{1}, R_{2}}\left(\left\|h_{1}-h_{2}\right\|_{\mathbb{E}_{1}(T)}+\left\|h_{0}^{1}-h_{0}^{2}\right\|_{X_{\gamma}}\right)+\left\|\tilde{v}_{0}^{1}-\tilde{v}_{0}^{2}\right\|_{Y_{\gamma}} .
\end{aligned}
$$

Now from (3.18) the claim follows.

As preparation for the proof of the local well-posedness for (2.6)-(2.13) we show that the term coming from the Navier-Stokes part in (2.12) is sufficiently regular:

Corollary 3.5 Let $p, q$ and $\varepsilon, T_{0}$ for $R>0$ as in Theorem 3.1. For $0<T \leq T_{0}, h \in V_{T, \varepsilon}$ and $v_{0} \in Y_{\gamma, h(0)} \cap L_{\sigma}^{q}(\Omega)$ with $\left\|v_{0}\right\|_{Y_{\gamma, h(0)}} \leq R$ for $j=1,2$ let

$$
G_{T}\left(h, v_{0}\right):=a_{1}(h) \cdot \operatorname{tr}_{\Sigma}\left(F_{T}\left(h, v_{0}\right)_{1}\right),
$$

where $a_{1}(h)$ is defined in Subsection 2.4 and $F_{T}\left(h, v_{0}\right)$ stems from Theorem 3.1. Then for a $s>p$ it holds $G_{T}\left(h, v_{0}\right) \in L^{s}\left(0, T ; X_{0}\right)$ and $\left\|G_{T}\left(h, v_{0}\right)\right\|_{L^{s}\left(0, T ; X_{0}\right)} \leq C(R)$ as well as

$$
\left.G_{T}\left(h, v_{0}\right)\right|_{[0, \tilde{T}]}=G_{\tilde{T}}\left(\left.h\right|_{[0, \tilde{T}]}, v_{0}\right) \quad \text { for all } 0<\tilde{T} \leq T .
$$

Moreover, for $h_{j}, h_{0}^{j}:=h_{j}(0), v_{0}^{j}$ and $\tilde{v}_{0}^{j}:=v_{0}^{j} \circ \tilde{\Theta}_{h_{0}^{j}}, j=1,2$ as in Theorem 3.1 it holds $\left\|G_{T}\left(h_{1}, v_{0}^{1}\right)-G_{T}\left(h_{2}, v_{0}^{2}\right)\right\|_{L^{s}\left(0, T ; X_{0}\right)} \leq C_{R}\left(\left\|h_{1}-h_{2}\right\|_{\mathbb{E}_{1}(T)}+\left\|h_{0}^{1}-h_{0}^{2}\right\|_{X_{\gamma}}+\left\|\tilde{v}_{0}^{1}-\tilde{v}_{0}^{2}\right\|_{Y_{\gamma}}\right)$.

Proof. From Remark 3.4 we know that $Y_{T}^{1} \rightarrow L^{s}\left(0, T ; X_{0}\right)^{n}: u \mapsto \operatorname{tr}_{\Sigma} u$ is bounded, linear for some $s>p$ and the operator norm is bounded independent of $T>0$ if we add $\|u(0)\|_{Y_{\gamma}}$ in the $Y_{T}^{1}$-norm. Hence $\operatorname{tr}_{\Sigma}\left(F_{T}\left(h, v_{0}\right)_{1}\right) \in L^{s}\left(0, T ; X_{0}\right)^{n}$ and from Theorem 3.1 and (3.16) we deduce $\left\|\operatorname{tr}_{\Sigma}\left(F_{T}\left(h, v_{0}\right)_{1}\right)\right\|_{L^{s}\left(0, T ; X_{0}\right)^{n}} \leq C(R)$ as well as

$$
\begin{aligned}
& \left\|\operatorname{tr}_{\Sigma}\left(F_{T}\left(h_{1}, v_{0}^{1}\right)_{1}\right)-\operatorname{tr}_{\Sigma}\left(F_{T}\left(h_{2}, v_{0}^{2}\right)_{1}\right)\right\|_{L^{s}\left(0, T ; X_{0}\right)^{n}} \leq \\
& \quad \leq C_{R}\left(\left\|h_{1}-h_{2}\right\|_{\mathbb{E}_{1}(T)}+\left\|h_{0}^{1}-h_{0}^{2}\right\|_{X_{\gamma}}+\left\|\tilde{v}_{0}^{1}-\tilde{v}_{0}^{2}\right\|_{Y_{\gamma}}\right) .
\end{aligned}
$$

Since pointwise multiplication is a product on $C^{1}(\Sigma) \times X_{0} \rightarrow X_{0}$, we obtain $G_{T}\left(h, v_{0}\right) \in$ $L^{s}\left(0, T ; X_{0}\right)$ with $\left\|G_{T}\left(h, v_{0}\right)\right\|_{L^{s}\left(0, T ; X_{0}\right)} \leq \tilde{C}(R)$ by Corollary 2.7 and (3.20) together with a null addition yields the estimate in the lemma. Equation (3.19) follows from the respective one for $F_{T}$ in Theorem 3.1 . 


\section{Local Well-Posedness}

\section{Local Well-Posedness}

In this section we show the local well-posedness for the transformed Navier-Stokes/mean curvature flow system (2.6)-(2.13). Let $p>n+2$ and $2<q<3$ with $1+\frac{n+2}{p}>\frac{n+2}{q}$. For $\varepsilon, T_{0}>0$ to $R>0$ as in Theorem 3.1 and $0<T \leq T_{0}$ as well as $h \in V_{T}^{p}, h_{0} \in X_{\gamma}$ with $\left\|h_{0}\right\|_{X_{\gamma}} \leq \varepsilon$ and $v_{0} \in Y_{\gamma, h(0)} \cap L_{\sigma}^{q}(\Omega)$ with $\left\|v_{0}\right\|_{Y_{\gamma, h(0)}} \leq R$ the system is equivalent to the abstract evolution equation

$$
\begin{aligned}
\partial_{t} h-G_{T}\left(h, v_{0}\right) & =a_{2}(h) K(h) & & \text { on } \Sigma \times(0, T), \\
\left.h\right|_{t=0} & =h_{0} & & \text { on } \Sigma,
\end{aligned}
$$

where $G_{T}$ is defined in Corollary 3.5. Here "equivalent" means that $(u, \tilde{q}, h) \in Y_{T} \times V_{T}$ solves (2.6)-(2.13) if and only if $h$ fulfils (4.1)-(4.2) and it holds $(u, \tilde{q})=F_{T}\left(h, v_{0}\right)$.

In order to show local well-posedness for (4.1)-(4.2), we use that by Lemma 2.8 the transformed mean curvature $K(h)$ has a quasilinear structure with respect to the height function $h$ and for $h=0$ the principal part is given by the Laplace-Beltrami operator $\Delta_{\Sigma}$. Here $\Delta_{\Sigma}: X_{1} \rightarrow X_{0}$ has maximal $L^{p}$-regularity on finite intervals because of Theorem 6.4.3 in Prüss and Simonett [18]. To give a simple proof for the local well-posedness, we proceed as in the case of quasilinear parabolic equations, compare e.g. Köhne, Prüss and Wilke [13], Theorem 2.1 (for $\mu=1$ there). We especially cannot apply this result directly since $G_{T}$ is a non-local operator. That means $G_{T}\left(h, v_{0}\right)$ at point $x_{0} \in \Sigma$ and time $t_{0} \in[0, T]$ in general depends on values $h(x, t)$ with $(x, t) \in \Sigma \times[0, T]$ outside a neighbourhood of $\left(x_{0}, t_{0}\right)$. Nevertheless we can essentially adapt the proof of [13] (for $\mu=1$ ) since by Corollary 3.5 we have that $G_{T}$ is sufficiently regular and compatible with restrictions in time on $[0, \tilde{T}]$ for $0<\tilde{T} \leq T$. Intuitively the velocity and pressure at time $T$ depend on the evolution of the entire interface from the beginning up to time $T$.

The result for the local well-posedness of the transformed Navier-Stokes/mean curvature flow system (2.6)-(2.13) reads as follows:

Theorem 4.1 Let $p>n+2$ and $2<q<3$ with $1+\frac{n+2}{p}>\frac{n+2}{q}$. For $R>0$ there exist $T_{0}=T_{0}(R), \varepsilon_{0}=\varepsilon_{0}(R)>0$ such that for all $0<T \leq T_{0}$ and $h_{0} \in X_{\gamma}$ with $\left\|h_{0}\right\|_{X_{\gamma}} \leq \varepsilon_{0}$ and $v_{0} \in Y_{\gamma, h_{0}} \cap L_{\sigma}^{q}(\Omega)$ with $\left\|v_{0}\right\|_{Y_{\gamma, h_{0}}} \leq R$ there is a unique solution $(u, \tilde{q}, h)\left(h_{0}, v_{0}\right) \in Y_{T} \times V_{T}$ of (2.6) $-(2.13)$ and

$$
\left\|(u, \tilde{q}, h)\left(h_{0}^{1}, v_{0}^{1}\right)-(u, \tilde{q}, h)\left(h_{0}^{2}, v_{0}^{2}\right)\right\|_{Y_{T} \times V_{T}} \leq C_{R}\left(\left\|h_{0}^{1}-h_{0}^{2}\right\|_{X_{\gamma}}+\left\|\tilde{v}_{0}^{1}-\tilde{v}_{0}^{2}\right\|_{Y_{\gamma}}\right)
$$

holds for $h_{0}^{j} \in X_{\gamma}$ with $\left\|h_{0}^{j}\right\|_{X_{\gamma}} \leq \varepsilon_{0}$ and $v_{0}^{j} \in Y_{\gamma, h_{j}(0)} \cap L_{\sigma}^{q}(\Omega)$ with $\left\|v_{0}^{j}\right\|_{Y_{\gamma, h_{j}(0)}} \leq R$ as well as $\tilde{v}_{0}^{j}:=v_{0}^{j} \circ \tilde{\Theta}_{h_{0}^{j}}$ for $j=1,2$.

Proof. With $P, Q$ as in Lemma2.8 and $U_{0}$ as in Remark2.5 we denote $\tilde{B}(h):=a_{2}(h) P(h)$ and $\tilde{F}(h):=a_{2}(h) Q(h)$ for all $h \in U_{0}$. Because of Theorem 6.4.3 in Prüss and Simonett [18] there is a $w>0$ such that $\Delta_{\Sigma}-w I: X_{1} \rightarrow X_{0}$ has maximal $L^{p}$-regularity on $\mathbb{R}_{+}$. Therefore we consider $B:=\tilde{B}-w I$ and $F:=\tilde{F}+w I$. Since $a_{2}(0)=1$ we have $B(0)=\Delta_{\Sigma}-w I$. Moreover, Corollary 2.7 yields $B \in C^{1}\left(U_{0} ; \mathcal{L}\left(X_{1}, X_{0}\right)\right)$ and $F \in C^{1}\left(U_{0} ; X_{0}\right)$. The embedding $X_{\gamma} \hookrightarrow L^{\infty}(\Sigma)$ implies $\overline{B_{X_{\gamma}}\left(0, \delta_{0}\right)} \subseteq U_{0}$ for $0<\delta_{0}<R_{0}$ small. So if $\delta_{0}$ is sufficiently small, by the mean value theorem, there is a $L>0$ such 


\section{Local Well-Posedness}

that

$$
\begin{gathered}
\left\|B\left(h_{1}\right) v-B\left(h_{2}\right) v\right\|_{X_{0}} \leq L\left\|h_{1}-h_{2}\right\|_{X_{\gamma}}\|v\|_{X_{1}}, \\
\left\|F\left(h_{1}\right)-F\left(h_{2}\right)\right\|_{X_{0}} \leq L\left\|h_{1}-h_{2}\right\|_{X_{\gamma}}
\end{gathered}
$$

holds for all $h_{1}, h_{2} \in \overline{B_{X_{\gamma}}\left(0, \delta_{0}\right)}$ and $v \in X_{1}$.

Now fix $R>0$ and let $T_{0}=T_{0}(R), \varepsilon=\varepsilon(R)>0$ be as in Theorem 3.1. Then for $0<T \leq T_{0}$ and $h \in V_{T}$ as well as $h_{0} \in X_{\gamma}$ with $\left\|h_{0}\right\|_{X_{\gamma}} \leq \varepsilon$ and $v_{0} \in Y_{\gamma, h_{0}} \cap L_{\sigma}^{q}(\Omega)$ with $\left\|v_{0}\right\|_{Y_{\gamma, h_{0}}} \leq R$ the system (2.6)-(2.13) or (4.1)-(4.2), respectively, are equivalent to

$$
\begin{aligned}
\partial_{t} h-B(h) h & =F(h)+G_{T}\left(h, v_{0}\right) \quad \text { in }(0, T), \\
h(0) & =h_{0} .
\end{aligned}
$$

Here $B(h)$ and $F(h)$ are defined in the sense of $V_{T} \subseteq C^{0}\left([0, T] ; U_{0}\right)$ by Remark [2.5. In particular, it holds $B(h) \in C^{0}\left([0, T] ; \mathcal{L}\left(X_{1}, X_{0}\right)\right)$.

The idea for solving (4.4)-(4.5) is as follows: we fix $B(h)$ at $h=0$ and apply Banach's fixed-point theorem to $H\left(. ; h_{0}, v_{0}\right)$ where $H\left(h ; h_{0}, v_{0}\right):=f$ is the unique solution of

$$
\begin{aligned}
\partial_{t} f-B(0) f & =B(h) h-B(0) h+F(h)+G_{T}\left(h, v_{0}\right) \quad \text { in }(0, T), \\
f(0) & =h_{0} .
\end{aligned}
$$

The unique solvability of (4.6)-(4.7) will follow from Prüss and Simonett [18], Theorem 3.5.5 if we extend the right hand side by zero to $\mathbb{R}_{+}$. To this end we need suitable sets for the height function. For $0<\delta<\min \left\{\varepsilon, \delta_{0}\right\}, 0<r<R_{0}-\delta_{0}$ and $0<T \leq T_{0}$ we set

$$
\mathbb{B}_{r, T, h_{0}}:=\left\{h \in \mathbb{E}_{1}(T): h(0)=h_{0},\|h\|_{\mathbb{E}_{1}(T)} \leq r\right\} \quad \text { for } h_{0} \in \overline{B_{X_{\gamma}}(0, \delta)} .
$$

For $r, \delta>0$ small we show $\mathbb{B}_{r, T, h_{0}} \subseteq V_{T} \cap C^{0}\left([0, T] ; \overline{B_{X_{\gamma}}\left(0, \delta_{0}\right)}\right)$. Therefore let $h \in \mathbb{B}_{r, T, h_{0}}$.

Because of $\|h\|_{\mathbb{E}_{1}(T)}+\left\|h_{0}\right\|_{X_{\gamma}} \leq r+\delta<R_{0}$ and our choice of $\delta_{0}$ it suffices to show $\|h(t)\|_{X_{\gamma}} \leq \delta_{0}$ for all $t \in[0, T]$ if $r, \delta>0$ are small independent of $h$. By Prüss and Simonett [18], Theorem 3.5.5 there is a unique solution $h_{0}^{*} \in \mathbb{E}_{1}\left(\mathbb{R}_{+}\right)$of

$$
\partial_{t} h_{0}^{*}-B(0) h_{0}^{*}=0, \quad h_{0}^{*}(0)=h_{0} .
$$

Since $h(0)=h_{0}^{*}(0)$, we get from Theorem A.6 with a null addition

$$
\|h\|_{C^{0}\left([0, T] ; X_{\gamma}\right)} \leq C\left(\|h\|_{\mathbb{E}_{1}(T)}+\left\|h_{0}\right\|_{X_{\gamma}}\right) \leq c(r+\delta) .
$$

For $r, \delta>0$ small we obtain $\|h(t)\|_{X_{\gamma}} \leq \delta_{0}$ for all $t \in[0, T]$ and thus the above inclusion.

Now we can define $H\left(. ; h_{0}, v_{0}\right) \equiv H_{r, T}\left(. ; h_{0}, v_{0}\right): \mathbb{B}_{r, T, h_{0}} \rightarrow \mathbb{E}_{1}(T): h \mapsto f$ where $f$ is the unique solution to (4.6)-(4.7). Then $h \in \mathbb{B}_{r, T, h_{0}}$ is a solution of (4.4)-(4.5) if and only if $h$ is a fixed-point of $H\left(. ; h_{0}, v_{0}\right)$. To apply Banach's theorem we need the following:

For $r, \delta, T>0$ small, all $g_{j} \in \overline{B_{X_{\gamma}}(0, \delta)}$ and $h_{j} \in \mathbb{B}_{r, T, g_{j}}$ as well as $v_{0}^{j} \in Y_{\gamma, g_{j}} \cap L_{\sigma}^{q}(\Omega)$ with $\left\|v_{0}^{j}\right\|_{Y_{\gamma, g_{j}}} \leq R$ and $\tilde{v}_{0}^{j}:=v_{0}^{j} \circ \tilde{\Theta}_{g_{j}}$ for $j=1,2$ we will show the following inequality:

$\left\|H\left(h_{1} ; g_{1}, v_{0}^{1}\right)-H\left(h_{2} ; g_{2}, v_{0}^{2}\right)\right\|_{\mathbb{E}_{1}(T)} \leq \frac{1}{2}\left\|h_{1}-h_{2}\right\|_{\mathbb{E}_{1}(T)}+c_{R}\left(\left\|g_{1}-g_{2}\right\|_{X_{\gamma}}+\left\|\tilde{v}_{0}^{1}-\tilde{v}_{0}^{2}\right\|_{Y_{\gamma}}\right)$.

Moreover, for all $r>0$ there are $\delta(r), T(r)>0$ such that for all $0<\delta \leq \delta(r)$ and $0<T \leq T(r)$ the estimate $\left\|H\left(0 ; h_{0}, v_{0}\right)\right\|_{\mathbb{E}_{1}(T)} \leq \frac{r}{2}$ is valid, where $h_{0} \in \overline{B_{X_{\gamma}}(0, \delta)}$. 


\section{Local Well-Posedness}

Now let us prove this. From Prüss and Simonett [18, Theorem 3.5.5 we know that there exists a $C>0$ independent of $T>0$ such that

$$
\begin{aligned}
& \left\|H\left(h_{1} ; g_{1}, v_{0}^{1}\right)-H\left(h_{2} ; g_{2}, v_{0}^{2}\right)\right\|_{\mathbb{E}_{1}(T)} \leq C\left(\left\|g_{1}-g_{2}\right\|_{X_{\gamma}}+\left\|F\left(h_{1}\right)-F\left(h_{2}\right)\right\|_{\mathbb{E}_{0}(T)}+\right. \\
& \left.+\left\|\left(B\left(h_{1}\right)-B(0)\right)\left(h_{1}-h_{2}\right)+\left(B\left(h_{1}\right)-B\left(h_{2}\right)\right) h_{2}+G_{T}\left(h_{1}, v_{0}^{1}\right)-G_{T}\left(h_{2}, v_{0}^{2}\right)\right\|_{\mathbb{E}_{0}(T)}\right) .
\end{aligned}
$$

Here because of (4.3) it holds $\left\|F\left(h_{1}\right)-F\left(h_{2}\right)\right\|_{\mathbb{E}_{0}(T)} \leq L T^{\frac{1}{p}}\left\|h_{1}-h_{2}\right\|_{C^{0}\left([0, T] ; X_{\gamma}\right)}$ and with $g_{j}^{*}$ as in (4.8) for $g_{j}, j=1,2$ instead of $h_{0}$ we conclude from Theorem A.6

$$
\left\|h_{1}-h_{2}\right\|_{C^{0}\left([0, T] ; X_{\gamma}\right)} \leq c\left(\left\|h_{1}-h_{2}\right\|_{\mathbb{E}_{1}(T)}+\left\|g_{1}-g_{2}\right\|_{X_{\gamma}}\right) .
$$

Furthermore (4.3) together with (4.9) and (4.10) implies

$$
\begin{aligned}
& \left\|\left(B\left(h_{1}\right)-B(0)\right)\left(h_{1}-h_{2}\right)+\left(B\left(h_{1}\right)-B\left(h_{2}\right)\right) h_{2}\right\|_{\mathbb{E}_{0}(T)} \leq \\
& \quad \leq L\left(\left\|h_{1}\right\|_{C^{0}\left([0, T] ; X_{\gamma}\right)}\left\|h_{1}-h_{2}\right\|_{\mathbb{E}_{1}(T)}+\left\|h_{1}-h_{2}\right\|_{C^{0}\left([0, T] ; X_{\gamma}\right)}\left\|h_{2}\right\|_{\mathbb{E}_{1}(T)}\right) \leq \\
& \quad \leq L C(r+\delta)\left\|h_{1}-h_{2}\right\|_{\mathbb{E}_{1}(T)}+L c r\left(\left\|h_{1}-h_{2}\right\|_{\mathbb{E}_{1}(T)}+\left\|g_{1}-g_{2}\right\|_{X_{\gamma}}\right) .
\end{aligned}
$$

Additionally, for some $s>p$ Corollary 3.5 and Hölder's inequality yield

$$
\left\|G_{T}\left(h_{1}, v_{0}^{1}\right)-G_{T}\left(h_{2}, v_{0}^{2}\right)\right\|_{\mathbb{E}_{0}(T)} \leq C_{R} T^{\frac{1}{p}-\frac{1}{s}}\left(\left\|h_{1}-h_{2}\right\|_{\mathbb{E}_{1}(T)}+\left\|g_{1}-g_{2}\right\|_{X_{\gamma}}+\left\|\tilde{v}_{0}^{1}-\tilde{v}_{0}^{2}\right\|_{Y_{\gamma}}\right) .
$$

For $r, \delta, T>0$ small we obtain the claimed difference estimate. It remains to treat $\left\|H\left(0 ; h_{0}, v_{0}\right)\right\|_{\mathbb{E}_{1}(T)}$. Theorem 3.5.5 in Prüss and Simonett [18] and Corollary [3.5] imply

$$
\left\|H\left(0 ; h_{0}, v_{0}\right)\right\|_{\mathbb{E}_{1}(T)} \leq C\left(\delta+\|F(0)\|_{\mathbb{E}_{0}(T)}+T^{\frac{1}{p}-\frac{1}{s}} C_{R}\right) .
$$

So if $\delta(r), T(r)>0$ are appropriate, we get $\left\|H\left(0 ; h_{0}, v_{0}\right)\right\|_{\mathbb{E}_{1}(T)} \leq \frac{r}{2}$ for $0<\delta \leq \delta(r)$ and $0<T \leq T(r)$. Altogether we have proven everything claimed above.

After this preparation we can choose $r, \delta, T_{0}>0$ small such that everything before holds for $r, \delta, T_{0}$ and for $2 r, \delta, T_{0}$. Then this also follows for $\tilde{r}, \delta, T$ where $\tilde{r} \in\{r, 2 r\}$ and $0<T \leq T_{0}$. Now we choose $h_{0}=g_{1}=g_{2} \in \overline{B_{X_{\gamma}}(0, \delta)}$ and $v_{0}=v_{0}^{1}=v_{0}^{2}$ above and obtain that $H_{\tilde{r}, T}\left(. ; h_{0}, v_{0}\right)$ is a strict contraction on $\mathbb{B}_{\tilde{r}, T, h_{0}}$ where the latter is a complete metric space as closed subset of a Banach space. Banach's theorem now yields a unique fixed-point $h_{\tilde{r}, T}^{*}\left(h_{0}, v_{0}\right) \in \mathbb{B}_{\tilde{r}, T, h_{0}}$ of $H_{\tilde{r}, T}\left(. ; h_{0}, v_{0}\right)$ in $\mathbb{B}_{\tilde{r}, T, h_{0}}$ which is a solution of (4.4)-(4.5) and

$$
\left(u^{*}, \tilde{q}^{*}, h^{*}\right)\left(h_{0}, v_{0}\right):=\left(F_{T}\left(h_{r, T}^{*}\left(h_{0}, v_{0}\right), v_{0}\right), h_{r, T}^{*}\left(h_{0}, v_{0}\right)\right) \in Y_{T} \times V_{T}
$$

solves the transformed Navier-Stokes/mean curvature flow system (2.6)-(2.13). The Lipschitz-dependence of $\left(u^{*}, \tilde{q}^{*}, h^{*}\right)$ on $\left(h_{0}, \tilde{v}_{0}\right)$ where $\tilde{v}_{0}:=v_{0} \circ \tilde{\Theta}_{h_{0}}$ is obtained as follows: For $h^{*}$ we use $H_{r, T}\left(h^{*}\left(h_{0}, v_{0}\right) ; h_{0}, v_{0}\right)=h^{*}\left(h_{0}, v_{0}\right)$ in the difference estimate proven before. Then for $\left(u^{*}, \tilde{q}^{*}\right)$ we utilize Theorem 3.1 ,

It remains to show uniqueness in $Y_{T} \times V_{T}$ for fixed $h_{0} \in \overline{B_{X_{\gamma}}(0, \delta)}$ and $v_{0}$ as in the theorem. In the following we write $h_{\tilde{r}, T}^{*}$ and $\left(u^{*}, \tilde{q}^{*}, h^{*}\right)$ instead of $h_{\tilde{r}, T}^{*}\left(h_{0}, v_{0}\right)$ and $\left(u^{*}, \tilde{q}^{*}, h^{*}\right)\left(h_{0}, v_{0}\right)$, respectively. In particular it holds $h^{*}=h_{r, T}^{*}$. We need that the fixed-point $h_{\tilde{r}, T}^{*}$ is the same for $\tilde{r} \in\{r, 2 r\}$ and remains the same up to restriction in time if we shrink $T$. Therefore let $0<\tilde{T} \leq T$ and $\tilde{r} \in\{r, 2 r\}$. For $h \in \mathbb{B}_{\tilde{r}, T, h_{0}}$ we have 


\section{A. Banach-Space-Valued Functions}

$\left.h\right|_{[0, \tilde{T}]} \in \mathbb{B}_{\tilde{r}, \tilde{T}, h_{0}}$ and because of Corollary 3.5 it holds $\left.G_{T}\left(h, v_{0}\right)\right|_{[0, \tilde{T}]}=G_{\tilde{T}}\left(\left.h\right|_{[0, \tilde{T}]}, v_{0}\right)$. Hence we obtain

$$
\left.H_{\tilde{r}, T}\left(h ; h_{0}, v_{0}\right)\right|_{[0, \tilde{T}]}=H_{\tilde{r}, \tilde{T}}\left(\left.h\right|_{[0, \tilde{T}]} ; h_{0}, v_{0}\right) .
$$

Since $H_{r, \tilde{T}}\left(h ; h_{0}, v_{0}\right)=H_{2 r, \tilde{T}}\left(h ; h_{0}, v_{0}\right)$ for all $h \in \mathbb{B}_{r, \tilde{T}, h_{0}} \subseteq \mathbb{B}_{2 r, \tilde{T}, h_{0}}$ the uniqueness of the respective fixed-point yields

$$
\left.h^{*}\right|_{[0, \tilde{T}]}=h_{r, \tilde{T}}^{*}=h_{2 r, \tilde{T}}^{*} \quad \text { for all } 0<\tilde{T} \leq T .
$$

Now the uniqueness in $Y_{T} \times V_{T}$ can be shown by a typical contradiction argument.

\section{A. Banach-Space-Valued Functions}

\section{A.1. Spaces of (Hölder-)Continuous Functions}

Let $X$ be a Banach space over $\mathbb{K}=\mathbb{R}$ or $\mathbb{C}$. For a closed interval $I \subseteq \mathbb{R}$ we denote by $C^{0}(I ; X), C_{b}^{0}(I ; X), B U C(I ; X)$ and $C^{0, \alpha}(I ; X)$ for $\alpha \in(0,1]$ the set of continuous; continuous and bounded; bounded and uniformly continuous; bounded and Höldercontinuous functions $f: I \rightarrow X$, respectively. In the latter case we write $[f]_{C^{0, \alpha}(I ; X)}$ for the semi-norm and we set $C^{\alpha}(I ; X):=C^{0, \alpha}(I ; X)$ if $\alpha \in(0,1)$. Moreover, let $C^{0,0}(I ; X):=C^{0}(I ; X)$. If $I \subseteq \mathbb{R}$ is an open interval, then $C^{\infty}(\bar{I} ; X)$ is the set of all smooth $f: I \rightarrow X$ such that $f$ and all derivatives can be extended continuously to $\bar{I}$ and $C_{0}^{\infty}(I ; X)$ denotes the set of all $f \in C^{\infty}(\bar{I} ; X)$ with compact support in $I$. For suitable product estimates we need:

Definition A.1 Let $X, Y, Z$ be Banach spaces over $\mathbb{K}=\mathbb{R}$ or $\mathbb{C}$. Then $B: X \times Y \rightarrow Z$ is called product if $B$ is bilinear and $\|B(f, g)\|_{Z} \leq C_{0}\|f\|_{X}\|g\|_{Y}$ holds for all $(f, g) \in X \times Y$ and some $C_{0}>0$.

With a null addition one can show that for any closed, bounded interval $I \subseteq \mathbb{R}$ and $\alpha \in[0,1]$ the product $B$ induces a product on the corresponding Hölder-spaces to the exponent $\alpha$ and in the estimate one can choose the same $C_{0}$. For estimates of nonlinear terms we use

Lemma A.2 Let $I \subseteq \mathbb{R}$ a closed, bounded interval, $n, m \in \mathbb{N}$ and $\Omega \subseteq \mathbb{R}^{n}$ open and bounded, $U \subseteq \mathbb{R}^{m}$ open, $F: U \rightarrow \mathbb{R}$ be $C^{1}$ and $K \subseteq U$ compact and convex. For $\alpha \in[0,1]$ let

$$
M:=\left\{u \in C^{0, \alpha}\left(I ; C^{0}\left(\bar{\Omega} ; \mathbb{R}^{m}\right)\right): u(t)(x) \in K \text { for all } t \in I, x \in \bar{\Omega}\right\}
$$

and $\tilde{F}(u)(t)(x):=F(u(t)(x))$ for $u \in M$ and $t \in I, x \in \bar{\Omega}$. Then for $u \in M$ with $\|u\|_{C^{0, \alpha}\left(I ; C^{0}(\bar{\Omega})^{m}\right)} \leq R$ it holds $\tilde{F}(u) \in C^{0, \alpha}\left(I ; C^{0}(\bar{\Omega})\right)$ with $\|\tilde{F}(u)\|_{C^{0, \alpha}\left(I ; C^{0}(\bar{\Omega})\right)} \leq C(R)$ where $C(R)>0$ is independent of $I$ and $\alpha$.

Moreover, if $F$ is $C^{2}$, then for all $u, v \in M$ with norm estimated by $R$ there is a $C(R)>0$ independent of $I$ and $\alpha$ such that $\|\tilde{F}(u)-\tilde{F}(v)\|_{C^{0, \alpha}\left(I ; C^{0}(\bar{\Omega})\right)} \leq C(R) \| u-$ $v \|_{C^{0, \alpha}\left(I ; C^{0}(\bar{\Omega})^{m}\right)^{*}}$ 


\section{A. Banach-Space-Valued Functions}

Proof. For $u, v \in M$ and $t \in I, x \in \bar{\Omega}$ the mean value theorem implies

$$
\tilde{F}(u(t)(x))-\tilde{F}(v(t)(x))=\int_{0}^{1} D F(s u(t)(x)+(1-s) v(t)(x)) d s \cdot(u(t)(x)-v(t)(x)) .
$$

Now the first part directly follows from the compactness of $K$. If additionally $F$ is $C^{2}$ we apply the first part to $G: U \times U \rightarrow \mathbb{R}:(\tilde{u}, \tilde{v}) \mapsto \int_{0}^{1} D F(s \tilde{u}+(1-s) \tilde{v}) d s$ and $K \times K$ instead of $K$ using a product estimate.

In the following lemma let all functions have values in $\mathbb{R}$.

Lemma A.3 Let $I \subseteq \mathbb{R}$ be a closed, bounded interval, $\Omega \subseteq \mathbb{R}^{n}$ be a bounded, connected domain, $\alpha \in[0,1]$ and $k=0,1$. For $\beta \in \mathbb{R}$ and $c_{0}>0$ we consider $F(h):=h^{\beta}$ acting on $U_{k}:=\left\{h \in C^{k}(\bar{\Omega}): h(x)>c_{0}\right.$ for all $\left.x \in \bar{\Omega}\right\}$ and on

$$
V_{\alpha, k}:=\left\{h \in C^{0, \alpha}\left(I ; C^{k}(\bar{\Omega})\right): h(t) \in U_{k} \text { for all } t \in I\right\},
$$

respectively. Then $F \in C^{1}\left(U_{k} ; C^{k}(\bar{\Omega})\right)$ and $F \in C^{1}\left(V_{\alpha, k} ; C^{0, \alpha}\left(I ; C^{k}(\bar{\Omega})\right)\right)$ and for $R>0$ arbitrary and all $h \in U_{k}$ and $h \in V_{\alpha, k}$ with norm less or equal $R$, respectively, it holds that $F(h)$ and $F^{\prime}(h)$ is bounded by a constant $C_{R, \beta, c_{0}}>0$ independent of $I$.

Proof. For $\beta \in \mathbb{N}_{0}$ the claim follows from the product rule. Therefore it is enough to consider the case $-\infty<\beta<1$. By scaling we can also assume $c_{0}=1$. Now one uses that for $\beta<1$ and $x, y \geq 1$ it holds that $\left|x^{\beta}-y^{\beta}\right| \leq c_{\beta}|x-y|$ and $x^{\beta} \leq C_{\beta}(1+|x|)$. Moreover, for $k=1$ we have $\partial_{x_{i}}(F(h))=\beta h^{\beta-1} \partial_{x_{i}} h$ for $i=1, \ldots, n$. From this the continuity of $F$ on respective spaces and the estimate for $F(h)$ follows. In both cases our candidate for the derivative is $G(h)(\rho):=\beta h^{\beta-1} \rho$ on corresponding spaces. Replacing $\beta-1$ by $\beta$ above one can show that $G$ is well-defined, continuous and satisfies the desired estimate for $F^{\prime}$ in the lemma. To verify the definition of the Fréchet-derivative one uses that for small $\rho$

$$
(F(h+\rho)-F(h)-G(h) \rho)=\int_{0}^{1} \int_{0}^{1} \beta(\beta-1)(h+u v \rho)^{\beta-2} u d v d u \rho^{2} .
$$

If $\rho$ is bounded by a small $\varepsilon>0$ in the respective norm, Lemma A.2 yields for $k=0$ that the first part can be estimated by $c(h, \varepsilon)$ in the corresponding norm. Then product estimates yield the case $k=0$ and the case $k=1$ follows from this by replacing $\beta-1$ by $\beta$ and using the identity for $\partial_{x_{i}}(F(h))$.

\section{A.2. Lebesgue-, Sobolev- and Besov-Spaces}

Let $I \subseteq \mathbb{R}$ be measurable, $X$ be a Banach space and $1 \leq p \leq \infty$. Then $L^{p}(I ; X)$ are the usual Bochner spaces and $W_{p}^{k}(I ; X)$ for $I$ open and $k \in \mathbb{N}$ are the $X$-valued Sobolev spaces.

Moreover, we need vector-valued variants of some fractional order Besov- and Sobolevspaces: Therefore let $s \in(0,1), 1 \leq p \leq \infty$ and $I$ be an interval in $\mathbb{R}$. Then we define $B_{p, \infty}^{s}(I ; X):=\left\{f \in L^{p}(I ; X):\|f\|_{B_{p, \infty}^{s}(I ; X)}<\infty\right\}$ where the norm is given by $\|f\|_{B_{p, \infty}^{s}(I ; X)}:=\|f\|_{L^{p}(I ; X)}+[f]_{B_{p, \infty}^{s}(I ; X)}$ with

$$
[f]_{B_{p, \infty}^{s}(I ; X)}:=\sup _{0<h \leq 1}\left\|\frac{f(.+h)-f}{h^{s}}\right\|_{L^{p}\left(I_{h} ; X\right)} \quad \text { and } I_{h}:=\{x \in I: x+h \in I\} .
$$




\section{A. Banach-Space-Valued Functions}

For $s \in(0,1)$ and $1 \leq p<\infty$ we set $W_{p}^{s}(I ; X):=\left\{f \in L^{p}(I ; X):\|f\|_{W_{p}^{s}(I ; X)}<\infty\right\}$ where the norm is given by $\|f\|_{W_{p}^{s}(I ; X)}^{p}:=\|f\|_{L^{p}(I ; X)}^{p}+[f]_{W_{p}^{s}(I ; X)}^{p}$ and

$$
[f]_{W_{p}^{s}(I ; X)}^{p}:=\int_{I} \int_{I}\left(\frac{\|f(x)-f(y)\|}{|x-y|^{s}}\right)^{p} \frac{d x d y}{|x-y|} .
$$

All the above spaces are Banach spaces since $L^{p}(I ; X)$ and $L^{1}(I \times I)$ are complete. The above definitions equal those in Simon [21] up to equivalent norms. Moreover, for $s>0$ and $1<p<\infty$ the Bessel-potential spaces are denoted by $H_{p}^{s}(I ; X)$.

Additionally, we need spaces with mixed regularity. Let $\Omega \subseteq \mathbb{R}^{n}$ be open, $r, s \in \mathbb{N}$, $1<p<\infty$ and $T>0$. Then we define $W_{p}^{r, s}\left(\Omega_{T}\right):=L^{p}\left(0, T ; W_{p}^{r}(\Omega)\right) \cap W_{p}^{s}\left(0, T ; L^{p}(\Omega)\right)$. If $T=\infty$ we write $\Omega \times \mathbb{R}_{+}$instead of $\Omega_{T}$. Further anisotropic spaces are defined analogously. For properties of such spaces cf. the appendix in Grubb [10] and Denk, Hieber and Prüss [6].

We need product estimates for Hölder spaces with suitable Sobolev- and Besov-spaces:

Lemma A.4 Let $X, Y, Z$ be Banach spaces over $\mathbb{K}=\mathbb{R}$ or $\mathbb{C}$ and $B: X \times Y \rightarrow Z$ be a product in sense of Definition A.1. Moreover, let $I \subseteq \mathbb{R}$ be a finite interval, $s \in(0,1)$ and $1 \leq p \leq \infty$. Then $C^{s}(\bar{I} ; X) \times B_{p, \infty}^{s}(I ; Y) \rightarrow B_{p, \infty}^{s}(I ; Z):(f, g) \mapsto B(f, g)$ is a product and in the product estimate one can choose the same constant as for $B$.

If $s \in(0,1), 1 \leq p<\infty$ and $\varepsilon>0$ is such that $s+\varepsilon<1$ holds, then $B$ induces a product on $C^{s+\varepsilon}(\bar{I} ; X) \times W_{p}^{s}(I ; Y) \rightarrow W_{p}^{s}(I ; Z)$ and the constant in the estimate can be chosen independent of $|I|$ if $|I| \leq T$ for a fixed $T>0$.

Proof. First one can show that $B$ induces a product on $C^{0}(\bar{I} ; X) \times L^{p}(I ; X) \rightarrow L^{p}(I ; X)$ and in the estimate the same constant can be chosen. Then the first part follows using a null addition. Now let $f \in C^{s+\varepsilon}(\bar{I} ; X)$ and $g \in W_{p}^{s}(I ; Y)$. Then a null addition yields

$$
[B(f, g)]_{W_{p}^{s}(I ; Z)}^{p} \leq C_{0}^{p}\left([f]_{C^{s+\varepsilon}(\bar{I} ; X)}^{p} \int_{I} \int_{I}\|g(x)\|_{Y}^{p} \frac{d x d y}{|x-y|^{1-\varepsilon p}}+\|f\|_{C^{0}(\bar{I} ; X)}^{p}[g]_{W_{p}^{s}(I ; Y)}^{p}\right) .
$$

By Tonelli and $I-x \subseteq[-|I|,|I|]$ the double integral can be estimated by $\|g\|_{L^{p}(I ; Y)}^{p} \frac{2|I|^{\varepsilon p}}{\varepsilon p}$.

The following lemma yields embeddings for suitable intersection spaces:

Theorem A.5 Let $X_{0}, X_{1}, X$ be Banach spaces over $\mathbb{K}=\mathbb{R}$ or $\mathbb{C}$ with $X_{1} \hookrightarrow X \hookrightarrow X_{0}$ and $I \subseteq \mathbb{R}$ be an open interval. Assume that for a $\theta \in(0,1)$ the following interpolation inequality holds: $\|x\|_{X} \leq C_{0}\|x\|_{X_{0}}^{1-\theta}\|x\|_{X_{1}}^{\theta}$ for all $x \in X_{0} \cap X_{1}$. Then

1. If $1 \leq p, p_{0}, p_{1} \leq \infty$ with $\frac{1}{p}=\frac{1-\theta}{p_{0}}+\frac{\theta}{p_{1}}$, then $L^{p_{0}}\left(I ; X_{0}\right) \cap L^{p_{1}}\left(I ; X_{1}\right) \hookrightarrow L^{p}(I ; X)$.

2. If $1 \leq p<\infty$, then $L^{p}\left(I ; X_{1}\right) \cap W_{p}^{1}\left(I ; X_{0}\right) \hookrightarrow B_{p, \infty}^{1-\theta}(I ; X)$.

In both cases analogous interpolation inequalities hold with $C_{0}$ and $3 C_{0}$, respectively.

Proof. The first part follows from Hölder's inequality. Now let $f \in L^{p}\left(I ; X_{1}\right) \cap W_{p}^{1}\left(I ; X_{0}\right)$ and $0<h \leq 1$ be arbitrary. It remains to estimate $[f]_{B_{p, \infty}^{1-\theta}(I ; X)}$. The first part yields

$$
\left\|\frac{f(.+h)-f}{h^{1-\theta}}\right\|_{L^{p}\left(I_{h} ; X\right)} \leq C_{0}\left\|\frac{f(.+h)-f}{h}\right\|_{L^{p}\left(I_{h} ; X_{0}\right)}^{1-\theta} 2^{\theta}\|f\|_{L^{p}\left(I ; X_{1}\right)}^{\theta} .
$$




\section{A. Banach-Space-Valued Functions}

Using $f(x+h)-f(x)=h \int_{0}^{1} f^{\prime}(x+t h) d t$, Hölder's inequality and Tonelli's theorem we get $\|f(.+h)-f\|_{L^{p}\left(I_{h} ; X_{0}\right)}^{p} \leq|h|^{p}\left\|f^{\prime}\right\|_{L^{p}\left(I ; X_{0}\right)}^{p}$. Thus the claim follows.

The following theorem characterizes the trace of functions in $\mathbb{E}_{1}(T)$ at $t=0$.

Theorem A.6 Let $X_{0}, X_{1}$ Banach spaces over $\mathbb{K}=\mathbb{R}$ or $\mathbb{C}$ with $X_{1} \hookrightarrow X_{0}$ and let $1<p<\infty$. Then for $\mathbb{E}_{1}\left(\mathbb{R}_{+}\right):=L^{p}\left(\mathbb{R}_{+} ; X_{1}\right) \cap W_{p}^{1}\left(0, \infty ; X_{0}\right)$ it holds that

$$
X_{\gamma}:=\left(X_{0}, X_{1}\right)_{1-\frac{1}{p}, p}=\left\{u(0): u \in \mathbb{E}_{1}\left(\mathbb{R}_{+}\right)\right\}
$$

and an equivalent norm on $X_{\gamma}$ is given by the induced quotient norm. Moreover we have $\mathbb{E}_{1}\left(\mathbb{R}_{+}\right) \hookrightarrow C_{b}^{0}\left(\mathbb{R}_{+} ; X_{\gamma}\right)$ and $\mathbb{E}_{1}(T) \hookrightarrow C^{0}\left([0, T] ; X_{\gamma}\right)$ for $0<T<\infty$ where the embedding constant is bounded independent of $T>0$ if we add $\|u(0)\|_{X_{\gamma}}$ in the $\mathbb{E}_{1}(T)$-norm. In particular this also holds for $u(0)=0$.

Proof. Up to $\mathbb{E}_{1}\left(\mathbb{R}_{+}\right) \hookrightarrow C_{b}^{0}\left(\mathbb{R}_{+} ; X_{\gamma}\right)$ this directly follows from the trace method, cf. Lunardi [17], Section 1.2. The embedding $\mathbb{E}_{1}(T) \hookrightarrow C^{0}\left([0, T] ; X_{\gamma}\right)$ can be shown by applying the one on $\mathbb{R}_{+}$to suitable extensions. E.g. one can first use reflection at $T$ and then multiply by a cutoff function. It remains to show the property of the embedding constant. In case $u(0)=0$ we extend $u$ as before and for $u(0) \neq 0$ we subtract an $\tilde{u} \in \mathbb{E}_{1}\left(\mathbb{R}_{+}\right)$with $\tilde{u}(0)=u(0)$ such that $\|\tilde{u}\|_{\mathbb{E}_{1}\left(\mathbb{R}_{+}\right)} \leq 2\|u(0)\|_{1-\frac{1}{p}, p}^{\text {tr }}$ holds and apply the first part to $u-\tilde{u}$.

By interpolation we get a whole scale of embeddings from Theorem A.6.

Theorem A.7 Let $X_{0}, X_{1}$ Banach spaces over $\mathbb{K}=\mathbb{R}$ or $\mathbb{C}$ with $X_{1} \hookrightarrow X_{0}$. Moreover, let $1<p<\infty$ and $T>0$. Then for $0<\theta<1-\frac{1}{p}$ and $1 \leq q \leq \infty$ it holds

$$
\mathbb{E}_{1}(T) \hookrightarrow C^{1-\theta-\frac{1}{p}}\left([0, T] ;\left(X_{0}, X_{1}\right)_{\theta, q}\right)
$$

and the embedding constant is bounded independent of $T>0$ if we add $\|u(0)\|_{X_{\gamma}}$ in the $\mathbb{E}_{1}(T)$-norm, where $\mathbb{E}_{1}(T)$ and $X_{\gamma}$ are as in Theorem A.6.

Proof. It is well-known that $W_{p}^{1}\left(0, T ; X_{0}\right) \hookrightarrow C^{0,1-\frac{1}{p}}\left([0, T] ; X_{0}\right)$ and the semi-norm is estimated by $\left\|u^{\prime}\right\|_{L^{p}\left(0, T ; X_{0}\right)}$ for all $u \in W_{p}^{1}\left(0, T ; X_{0}\right)$. Now one interpolates with the embedding from Theorem A.6 and uses the reiteration theorem in Lunardi [17], Corollary 1.24 and the interpolation inequality in [17], Corollary 1.7.

As another consequence of Theorem A.6 we obtain

Lemma A.8 Let $\Omega \subseteq \mathbb{R}^{n}, n \geq 2$ be a bounded $C^{\infty}$-domain and $\Sigma \subseteq \Omega$ be a connected, compact and smooth hypersurface that separates $\Omega$ in two disjoint, connected domains $\Omega^{ \pm}$with $\Sigma=\partial \Omega^{+}$. Moreover, let $0<T<\infty, 2 \leq p<\infty$ and $\Omega_{0}:=\Omega^{+} \cup \Omega^{-}$. Then

$$
\mathbb{E}_{1}(T):=L^{p}\left(0, T ; X_{1}\right) \cap W_{p}^{1}\left(0, T ; L^{p}(\Omega)\right) \hookrightarrow C^{0}\left([0, T] ; Y_{\gamma}\right)
$$

with $X_{1}:=W_{p}^{2}\left(\Omega_{0}\right) \cap W_{p, 0}^{1}(\Omega)$ and $Y_{\gamma}:=\left\{v \in L^{p}(\Omega):\left.v\right|_{\Omega^{ \pm}} \in W_{p}^{2-\frac{2}{p}}\left(\Omega^{ \pm}\right)\right\} \cap W_{p, 0}^{1}(\Omega)$. The embedding constant is bounded independent of $T>0$ if we add $\|u(0)\|_{Y_{\gamma}}$ in the norm. 
Proof. We define $X_{\gamma}:=\left(L^{p}(\Omega), X_{1}\right)_{1-\frac{1}{p}, p}$ and $\tilde{X}_{\gamma}:=\left(L^{p}(\Omega), W_{p}^{2}\left(\Omega_{0}\right)\right)_{1-\frac{1}{p}, p}$. Then TheoremA.6implies $\mathbb{E}_{1}(T) \hookrightarrow C^{0}\left([0, T] ; X_{\gamma}\right)$ and $\tilde{\mathbb{E}}_{1}(T):=L^{p}\left(0, T ; W_{p}^{2}\left(\Omega_{0}\right)\right) \cap W_{p}^{1}\left(0, T ; L^{p}(\Omega)\right)$ embeds to $C^{0}\left([0, T] ; \tilde{X}_{\gamma}\right)$. The embedding constants are bounded in a similar way as in the theorem. E.g. from the $K$-method, cf. Lunardi [17], Section 1.1 we obtain

$$
\tilde{X}_{\gamma}=\left\{v \in L^{p}(\Omega):\left.v\right|_{\Omega^{ \pm}} \in W_{p}^{2-\frac{2}{p}}\left(\Omega^{ \pm}\right)\right\}
$$

with equivalent norms. Hence it remains to show that for the continuous representative of $u \in \mathbb{E}_{1}(T)$ additionally $u(t) \in W_{p, 0}^{1}(\Omega)$ for all $t \in[0, T]$ holds. Therefore we use the characterization in Theorem A.6 to conclude $X_{\gamma} \hookrightarrow \tilde{X}_{\gamma}$. Here $X_{1}$ is dense in $X_{\gamma}$ by Lunardi [17, Proposition 1.17. For $v \in X_{1}$ it holds $\llbracket v \rrbracket=\operatorname{tr}_{\Sigma}\left(\left.v\right|_{\Omega^{-}}\right)-\operatorname{tr}_{\Sigma}\left(\left.v\right|_{\Omega^{+}}\right)=0$ and $\operatorname{tr}_{\partial \Omega} v=0$. By density this carries over to all $v \in X_{\gamma}$ because of $X_{\gamma} \hookrightarrow \tilde{X}_{\gamma} \hookrightarrow W_{p}^{1}\left(\Omega_{0}\right)$ for $2 \leq p<\infty$ and the continuity of the respective boundary trace operators. Using the definition of weak derivative and partial integration as well as the trace characterization of $W_{p, 0}^{1}(\Omega)$ we get $X_{\gamma} \subseteq \tilde{X}_{\gamma} \cap W_{p, 0}^{1}(\Omega)=Y_{\gamma}$.

Finally, we need some properties of certain types of intersection spaces:

Lemma A.9 Let $\Omega \subseteq \mathbb{R}^{n}$ a bounded, connected domain, $0<T \leq T_{0}$ and $1 \leq p<\infty$. Then $W_{T}:=C^{0}\left([0, T] ; C^{0}(\bar{\Omega})\right) \cap W_{p}^{1}\left(0, T ; L^{p}(\Omega)\right)$ is an algebra with pointwise multiplication and it holds $\|f g\|_{W_{T}} \leq C\|f\|_{W_{T}}\|g\|_{W_{T}}$ with $C>0$ independent of $T, T_{0}$ and $p$.

Moreover, for $f \in W_{T}$ with $f(t)(x) \geq c_{0}>0$ for all $(x, t) \in \bar{\Omega} \times[0, T]$ also $1 / f \in W_{T}$ and we have $\partial_{t}(1 / f)=-\partial_{t} f / f^{2}$. If additionally $\|f\|_{W_{T}} \leq R$, then $\left\|\frac{1}{f}\right\|_{W_{T}} \leq c_{R}$ with $c_{R}>0$ independent of $0<T \leq T_{0}$.

Proof. Using convolution one can show that $C^{\infty}\left([0, T] ; C^{0}(\bar{\Omega})\right)$ is dense in $W_{T}$. With this one can directly prove the first part and that a product rule holds. The second part follows by density utilizing Lemma A.3 and that the evaluation at any point in $\bar{\Omega}$ gives a bounded, linear functional on $C^{0}(\bar{\Omega})$.

\section{B. Maximal regularity for a two-phase Stokes system}

Let $2 \leq q<3, \Omega \subseteq \mathbb{R}^{n}, n \geq 2$ be a bounded $C^{\infty}$-domain and $\Sigma \subseteq \Omega$ a compact, smooth and connected hypersurface that divides $\Omega$ into two disjoint and connected domains $\Omega^{ \pm}$ with $\Sigma=\partial \Omega^{+}$and outer unit normal $\nu_{\Sigma}$. We define $\Omega_{0}:=\Omega^{+} \cup \Omega^{-}$and consider the following two-phase Stokes system

$$
\begin{aligned}
\partial_{t} u-\mu^{ \pm} \Delta u+\nabla \tilde{q} & =f & & \text { in } \Omega^{ \pm} \times(0, T), \\
\operatorname{div} u & =g & & \text { in } \Omega^{ \pm} \times(0, T), \\
\llbracket u \rrbracket & =0 & & \text { on } \Sigma \times(0, T), \\
\llbracket T(u, \tilde{q}) \rrbracket \nu_{\Sigma} & =a & & \text { on } \Sigma \times(0, T), \\
\left.u\right|_{\partial \Omega} & =0 & & \text { on } \partial \Omega \times(0, T), \\
\left.u\right|_{t=0} & =u_{0} & & \text { in } \Omega,
\end{aligned}
$$


where $\mu^{ \pm}>0$ and $T(u, \tilde{q}):=2 \mu^{ \pm} \operatorname{sym}(\nabla u)-\tilde{q} I$ in $\Omega^{ \pm}$. With the aid of Shimizu [20] we show an optimal existence result in an $L^{q}$-setting: For $(u, \tilde{q})$ we introduce the space $Y_{T}:=Y_{T}^{1} \times Y_{T}^{2}$ where

$$
\begin{aligned}
& Y_{T}^{1}:=W_{q}^{1}\left(0, T ; L^{q}(\Omega)\right)^{n} \cap L^{q}\left(0, T ; W_{q}^{2}\left(\Omega_{0}\right) \cap W_{q, 0}^{1}(\Omega)\right)^{n}, \\
& Y_{T}^{2}:=\left\{\tilde{q} \in L^{q}\left(0, T ; W_{q,(0)}^{1}\left(\Omega_{0}\right)\right): \llbracket \tilde{q} \rrbracket \in W_{q}^{1-\frac{1}{q}, \frac{1}{2}\left(1-\frac{1}{q}\right)}\left(\Sigma_{T}\right)\right\} .
\end{aligned}
$$

Here we require mean value 0 for $\tilde{q}$ in order to get uniqueness later. This implies necessary conditions for the data $\left(f, g, a, u_{0}\right)$ if (B.1)-(B.6) hold:

Lemma B.1 Let $2 \leq q<3, T>0$ and $(u, \tilde{q}) \in Y_{T}$. Then $\left(f, g, a, u_{0}\right)$ defined by (B.1) $-\left(\right.$ B.6) is contained in $\tilde{Z}_{T}:=\tilde{Z}_{T}^{1} \times \tilde{Z}_{T}^{2} \times\left(\tilde{Z}_{T}^{3}\right)^{n} \times Y_{\gamma}$ with $\tilde{Z}_{T}^{1}:=L^{q}\left(0, T ; L^{q}(\Omega)\right)^{n}$ and

$$
\begin{aligned}
& \tilde{Z}_{T}^{2}:=\left\{g \in L^{q}\left(0, T ; W_{q,(0)}^{1}\left(\Omega_{0}\right)\right) \cap W_{q}^{1}\left(0, T ; W_{q,(0)}^{-1}(\Omega)\right): \operatorname{tr}_{\Sigma}\left(\left.g\right|_{\Omega^{+}}\right) \in \tilde{Z}_{T}^{3}\right\} \\
& \tilde{Z}_{T}^{3}:=W_{q}^{1-\frac{1}{q}, \frac{1}{2}\left(1-\frac{1}{q}\right)}\left(\Sigma_{T}\right), \quad Y_{\gamma}:=\left\{v \in L^{q}(\Omega):\left.v\right|_{\Omega^{ \pm}} \in W_{q}^{2-\frac{2}{q}}\left(\Omega^{ \pm}\right)\right\}^{n} \cap W_{q, 0}^{1}(\Omega)^{n} .
\end{aligned}
$$

Additionally, the compatibility condition $\operatorname{div} u_{0}=\left.g\right|_{t=0}$ in $W_{q,(0)}^{-1}(\Omega)$ is valid and we have $\left\|\left(f, g, a, u_{0}\right)\right\|_{\tilde{Z}_{T}} \leq C\left(\|(u, \tilde{q})\|_{Y_{T}}+\left\|u_{0}\right\|_{Y_{\gamma}}\right)$ with $C>0$ independent of $(u, \tilde{q})$ and $T>0$.

Remark B.2 The spaces are equipped with the natural norms. In particular we include the term $\|\llbracket \tilde{q} \rrbracket\|_{\tilde{Z}_{T}^{3}}$ in the $Y_{T}^{2}$-norm and $\left\|\operatorname{tr}_{\Sigma}\left(\left.g\right|_{\Omega^{+}}\right)\right\|_{\tilde{Z}_{T}^{3}}$ in the $\tilde{Z}_{T^{2}}^{2}$-norm. One can directly verify that all spaces are Banach spaces.

Proof of Lemma B.1. The assertion for $f$ and $L^{q}$-in-time properties of $g$ can be directly shown. Lemma A.8 implies $u_{0} \in Y_{\gamma}$. Weak differentiability of $g$ follows from

$$
\int_{\Omega} g(t) \phi d x=-\int_{\Omega} u(t) \cdot \nabla \phi d x
$$

for all $\phi \in W_{q^{\prime}}^{1}(\Omega)$ and almost all $t \in(0, T)$. The latter identity also implies the compatibility condition. Results on mixed order Sobolev spaces yield together with $\llbracket \tilde{q} \rrbracket \in \tilde{Z}_{T}^{3}$ that $a, \operatorname{tr}_{\Sigma}\left(\left.g\right|_{\Omega^{+}}\right) \in \tilde{Z}_{T}^{3}$ and the uniform estimate in the lemma can be shown by extending functions in time with suitable operators, cf. proof of Lemma 3.3, below (3.14).

Our maximal regularity result for (B.1)-(B.6) is

Theorem B.3 Let $2 \leq q<3$ and $0<T \leq T_{0}$. Let $\left(f, g, a, u_{0}\right)$ be contained in the space $Z_{T}:=\left\{\left(f, g, a, u_{0}\right) \in \tilde{Z}_{T}: \operatorname{div} u_{0}=\left.g\right|_{t=0}\right.$ in $\left.W_{q,(0)}^{-1}(\Omega)\right\}$. Then the Stokes system (B.1) -(B.6) has exactly one solution $(u, \tilde{q})$ in $Y_{T}$. Moreover, there is a $C>0$ independent of $\left(f, g, a, u_{0}\right)$ and $0<T \leq T_{0}$ such that $\|(u, \tilde{q})\|_{Y_{T}} \leq C\left\|\left(f, g, a, u_{0}\right)\right\|_{\tilde{Z}_{T}}$.

Remark B.4 1. Further necessary conditions are $\operatorname{tr}_{\partial \Omega} g \in W_{q}^{1-\frac{1}{q}, \frac{1}{2}\left(1-\frac{1}{q}\right)}\left(\partial \Omega_{T}\right)$ and $\operatorname{tr}_{\Sigma}\left(\left.g\right|_{\Omega^{-}}\right) \in \tilde{Z}_{T}^{3}$. But these follow indirectly from the proof of Theorem B.3.

2. For simplicity we restricted to $2 \leq q<3$. In principle also for other scales of $q$ statements are possible. But then e.g. other necessary conditions can arise. For instance, if $q>3$ another compatibility condition appears involving the jump of the stress tensor, cf. Shimizu [20]. 


\section{B. Maximal Regularity for a two-Phase Stokes System}

Proof of Theorem B.3. The case $\left(a, g, u_{0}\right)=0$ follows from the announced result by Shimizu [20, Theorem 2. The main step for the proof is the investigation of model problems. For $\mathbb{R}^{n}$ with $\mathbb{R}^{n-1}$ as interface cf. Shibata and Shimizu [19]. For simplicity we do not give a proof of Theorem 2 in 20 . But we reduce the general case to the above one similar to A. and Wilke [2], proof of Theorem A.1.

First of all, we reduce to the case $\left.\left(g, u_{0}\right)\right|_{\Omega^{+}}=0$. Therefore we consider

$$
\begin{array}{ll}
\operatorname{div} v^{+}=\left.g\right|_{\Omega^{+}} & \text {in } \Omega^{+} \times(0, T), \\
\left.v^{+}\right|_{t=0}=\left.u_{0}\right|_{\Omega^{+}} & \text {in } \Omega^{+}
\end{array}
$$

and apply Theorem 1.1 in A. [1] (for $\Gamma_{1}=\emptyset, \Gamma_{2}=\Sigma$ and $f, a=0$ there). To this end we have to show the properties of $\left.g\right|_{\Omega^{+}}$and $\left.u_{0}\right|_{\Omega^{+}}$. First it holds

$$
\left.g\right|_{\Omega^{+}} \in L^{q}\left(0, T ; W_{q}^{1}\left(\Omega^{+}\right)\right) \quad \text { and } \quad \operatorname{tr}_{\Sigma}\left(\left.g\right|_{\Omega^{+}}\right) \in W_{q}^{1-\frac{1}{q}, \frac{1}{2}\left(1-\frac{1}{q}\right)}\left(\Sigma_{T}\right)
$$

by definition of $\tilde{Z}_{T}^{2}$. Moreover, for $w \in L_{(0)}^{q}(\Omega)$ and $\phi \in W_{q^{\prime}, 0}^{1}\left(\Omega^{+}\right)$

$$
\left\langle\left. w\right|_{\Omega^{+}}, \phi\right\rangle_{W_{q}^{-1}\left(\Omega^{+}\right), W_{q^{\prime}, 0}^{1}\left(\Omega^{+}\right)}=\int_{\Omega^{+}} w \phi d x=\int_{\Omega} w \tilde{e}_{0} \phi d x=\left\langle w, \tilde{e}_{0} \phi\right\rangle_{W_{q,(0)}^{-1}(\Omega), W_{q^{\prime},(0)}^{1}(\Omega)},
$$

where $e_{0} \phi \in W_{q^{\prime}, 0}^{1}(\Omega)$ is the extension by zero of $\phi$ and $\tilde{e}_{0} \phi:=e_{0} \phi-\frac{1}{|\Omega|} \int_{\Omega}\left(e_{0} \phi\right)(y) d y$. In particular this is also valid for $g(t)$ instead of $w$ for almost all $t \in(0, T)$. Since

$$
W_{q,(0)}^{-1}(\Omega) \rightarrow W_{q}^{-1}\left(\Omega^{+}\right): w \mapsto\left[\phi \mapsto\left\langle w, \tilde{e}_{0} \phi\right\rangle\right]
$$

is bounded and linear, we obtain $\left.g\right|_{\Omega^{+}} \in W_{q}^{1}\left(0, T ; W_{q}^{-1}\left(\Omega^{+}\right)\right)$, the norm is estimated by $C\|g\|_{\tilde{Z}_{T}^{2}}$ and for all $\phi \in W_{q^{\prime}, 0}^{1}\left(\Omega^{+}\right)$we have

$$
\left\langle\left.\left(\left.g\right|_{\Omega^{+}}\right)\right|_{t=0}, \phi\right\rangle_{W_{q}^{-1}\left(\Omega^{+}\right), W_{q^{\prime}, 0}^{1}\left(\Omega^{+}\right)}=\left\langle\left. g\right|_{t=0}, \tilde{e}_{0} \phi\right\rangle_{W_{q,(0)}^{-1}(\Omega), W_{q^{\prime},(0)}^{1}(\Omega)} .
$$

This yields $\operatorname{div}\left(\left.u_{0}\right|_{\Omega^{+}}\right)=\left.\left(\left.g\right|_{\Omega^{+}}\right)\right|_{t=0}$ in $W_{q}^{-1}\left(\Omega^{+}\right)$because of $u_{0} \in Y_{\gamma}$ and the compatibility condition in $Z_{T}$. Now Theorem 1.1 in A. [1] yields a solution $v^{+} \in W_{q}^{2,1}\left(\Omega_{T}^{+}\right)^{n}$ of (B.7)(B.8) with

$$
\left\|v^{+}\right\|_{W_{q}^{2,1}\left(\Omega_{T}^{+}\right)^{n}} \leq C_{T_{0}}\left(\|g\|_{\tilde{Z}_{T}^{2}}+\left\|u_{0}\right\|_{Y_{\gamma}}\right)
$$

where $C_{T_{0}}>0$ is independent of $0<T \leq T_{0}$. We extend $v^{+}$to $\tilde{v}^{+} \in Y_{T}^{1}$ using a suitable extension operator. Because of Lemma B.1 we have reduced to the case $\left.\left(g, u_{0}\right)\right|_{\Omega^{+}}=0$.

Now we want to trace the latter one back to the case $\left(g, u_{0}\right)=0$. Therefore we look at

$$
\begin{aligned}
\operatorname{div} v^{-} & =\left.g\right|_{\Omega^{-}} & & \text {in } \Omega^{-} \times(0, T), \\
\left.v^{-}\right|_{\partial \Omega^{-}} & =0 & & \text { on } \partial \Omega^{-} \times(0, T), \\
\left.v^{-}\right|_{t=0} & =\left.u_{0}\right|_{\Omega^{-}} & & \text {in } \Omega^{-}
\end{aligned}
$$

and again apply Theorem 1.1 in A. [1] (for $\Gamma_{1}=\partial \Omega^{-}, \Gamma_{2}=\emptyset$ and $f, a=0$ there). Since $\left.g\right|_{\Omega^{+}}=0$ it holds $\left.g\right|_{\Omega^{-}} \in L^{q}\left(0, T ; W_{q,(0)}^{1}\left(\Omega^{-}\right)\right)$. Furthermore, for $w \in L_{(0)}^{q}(\Omega)$ with $\left.w\right|_{\Omega^{+}}=0$ and all $\phi \in W_{q^{\prime}}^{1}\left(\Omega^{-}\right)$we have

$$
\left\langle\left. w\right|_{\Omega^{-}}, \phi\right\rangle_{W_{q^{\prime}}^{1}\left(\Omega^{-}\right)^{\prime}, W_{q^{\prime}}^{1}\left(\Omega^{-}\right)}=\int_{\Omega^{-}} w \phi d x=\int_{\Omega} w \tilde{E} \phi d x=\langle w, \tilde{E} \phi\rangle_{W_{q,(0)}^{-1}(\Omega), W_{q^{\prime},(0)}^{1}(\Omega)},
$$


where $E: W_{q^{\prime}}^{1}\left(\Omega^{-}\right) \rightarrow W_{q^{\prime}}^{1}(\Omega)$ is an extension operator and $\tilde{E} \phi:=E \phi-\frac{1}{|\Omega|} \int_{\Omega}(E \phi)(y) d y$. Since

$$
W_{q,(0)}^{-1}(\Omega) \rightarrow W_{q^{\prime}}^{1}\left(\Omega^{-}\right)^{\prime}: w \mapsto[\phi \mapsto\langle w, \tilde{E} \phi\rangle]
$$

is bounded and linear, we obtain $\left.g\right|_{\Omega^{-}} \in W_{q}^{1}\left(0, T ; W_{q^{\prime}}^{1}\left(\Omega^{-}\right)^{\prime}\right)$, the norm is estimated by $C\|g\|_{\tilde{Z}_{T}^{2}}$ and for all $\phi \in W_{q^{\prime}}^{1}\left(\Omega^{-}\right)$it holds that

$$
\left\langle\left.\left(\left.g\right|_{\Omega^{-}}\right)\right|_{t=0}, \phi\right\rangle_{W_{q^{\prime}}^{1}\left(\Omega^{-}\right)^{\prime}, W_{q^{\prime}}^{1}\left(\Omega^{-}\right)}=\left\langle\left. g\right|_{t=0}, \tilde{E} \phi\right\rangle_{W_{q,(0)}^{-1}(\Omega), W_{q^{\prime},(0)}^{1}(\Omega)} .
$$

Since $\left.u_{0}\right|_{\Omega^{+}}=0$ and $u_{0} \in Y_{\gamma}$ it follows that $\operatorname{div}\left(\left.u_{0}\right|_{\Omega^{-}}\right)=\left.\left(\left.g\right|_{\Omega^{-}}\right)\right|_{t=0}$ in $W_{q^{\prime}}^{1}\left(\Omega^{-}\right)^{\prime}$ with the compatibility condition in $Z_{T}$. By Theorem 1.1 in A. [1] there exists a solution $v^{-} \in W_{q}^{2,1}\left(\Omega_{T}^{-}\right)^{n}$ of (B.9)-(B.11) such that

$$
\left\|v^{-}\right\|_{W_{q}^{2,1}\left(\Omega_{T}^{-}\right)^{n}} \leq C_{T_{0}}\left(\|g\|_{\tilde{Z}_{T}^{2}}+\left\|u_{0}\right\|_{Y_{\gamma}}\right)
$$

where $C_{T_{0}}>0$ is independent of $0<T \leq T_{0}$. Because of $\left.v^{-}\right|_{\partial \Omega^{-}}=0$, we can extend $v^{-}$ by zero to $e_{0}\left(v^{-}\right) \in Y_{T}^{1}$. Lemma B.1 implies that we have reduced to the case $\left(g, u_{0}\right)=0$.

To trace this one back to the case $\left(g, a, u_{0}\right)=0$ we define for any $a: \Sigma \rightarrow \mathbb{R}^{n}$ the normal and tangential component $a_{\nu}:=a \cdot \nu_{\Sigma} \nu_{\Sigma}$ and $a_{\tau}:=a-a_{\nu}$, respectively. First we reduce to the case $\left(g, a_{\tau}, u_{0}\right)=0$. As in A. [1], proof of Lemma 2.5, 2. there is an $A \in W_{q}^{2,1}\left(\Omega_{T}^{+}\right)^{n}$ such that $\|A\|_{W_{q}^{2,1}\left(\Omega_{T}^{+}\right)^{n}} \leq C\|a\|_{\left(\tilde{Z}_{T}^{3}\right)^{n}}$ as well as

$$
\left.A\right|_{t=0}=0,\left.\quad A\right|_{\Sigma}=0, \quad\left(\left.2 \mu^{+} \operatorname{sym}(\nabla A)\right|_{\Sigma} \nu_{\Sigma}\right)_{\tau}=a_{\tau} \quad \text { and }\left.\quad(\operatorname{div} A)\right|_{\Sigma}=0
$$

But we also want $A$ to be divergence free. To this end one shows as in the proof of Lemma B.1 using $\left.A\right|_{\Sigma}=0$ that $\operatorname{div} A \in L^{q}\left(0, T ; W_{q, 0}^{1}\left(\Omega^{+}\right)\right) \cap W_{q}^{1}\left(0, T ; W_{q^{\prime}}^{1}\left(\Omega^{+}\right)^{\prime}\right)$ and the norm is estimated by $C\|A\|_{W_{q}^{2,1}\left(\Omega^{+}\right)^{n}}$. Now we apply the Bogovskiu-operator $B$ to $\operatorname{div} A$, cf. Geissert, Heck and Hieber [9], Theorem 2.5, and obtain

$$
B(\operatorname{div} A) \in L^{q}\left(0, T ; W_{q, 0}^{2}\left(\Omega^{+}\right)\right)^{n} \cap W_{q}^{1}\left(0, T ; L^{q}\left(\Omega^{+}\right)\right)^{n} \quad \text { with } \quad \operatorname{div}(B(\operatorname{div} A))=\operatorname{div} A,
$$

since $\operatorname{div} A$ has mean value 0 . Additionally, the norm of $B(\operatorname{div} A)$ is estimated by $C\|A\|_{W_{q}^{2,1}\left(\Omega^{+}\right)^{n}}$. Therefore the extension by zero to $\Omega$ of $\tilde{A}:=A-B(\operatorname{div} A)$ has the desired properties and we reduced to the case $\left(g, a_{\tau}, u_{0}\right)=0$.

Finally, to reduce the latter one to the case $\left(g, a, u_{0}\right)=0$, we subtract a suitable extension of $a \cdot \nu_{\Sigma} \in \tilde{Z}_{T}^{3}$ from the pressure: By the trace theorem there is a

$$
p^{+} \in L^{q}\left(0, T ; W_{q}^{1}\left(\Omega^{+}\right)\right) \quad \text { with }\left\|p^{+}\right\|_{L^{q}\left(0, T ; W_{q}^{1}\left(\Omega^{+}\right)\right)} \leq C\|a\|_{\left(\tilde{Z}_{T}^{3}\right)^{n}} \quad \text { and }\left.\quad p^{+}\right|_{\Sigma}=a \cdot \nu_{\Sigma} \text {. }
$$

We extend $p^{+}$by 0 to $\Omega$ and subtract the mean value which does not alter the jump. Hence we obtain a $\tilde{p}^{+} \in Y_{T}^{2}$ with $\left\|\tilde{p}^{+}\right\|_{Y_{T}^{2}} \leq C\|a\|_{\left(\tilde{Z}_{T}^{3}\right)^{n}}$ and $-\llbracket \tilde{p}^{+} \rrbracket \nu_{\Sigma}=a_{\nu}$.

Altogether we reduced the general case to the case $\left(g, a, u_{0}\right)=0$. 


\section{REFERENCES}

\section{References}

[1] H. Abels. Nonstationary stokes system with variable viscosity in bounded and unbounded domains. Discrete Contin. Dyn. Syst. Ser. S, 3(2):141-157, 2010.

[2] H. Abels and M. Wilke. Well-posedness and qualitative behaviour of solutions for a two-phase Navier-Stokes-Mullins-Sekerka system. Interfaces and Free Boundaries, 15:39-75, 2013.

[3] R. A. Adams and J. J. F. Fournier. Sobolev Spaces. Elsevier Ltd., second edition edition, 2003.

[4] H. Amann. Quasilinear parabolic problems via maximal regularity. Adv. Differential Equations, 10:1081-1110, 2005.

[5] J. Bergh and J. Löfström. Interpolation Spaces. Springer, Berlin - Heidelberg - New York, 1976

[6] R. Denk, M. Hieber, and J. Prüss. Optimal $L^{p}-L^{q}$-estimates for parabolic boundary value problems with inhomogeneous data. Math. Z., 257:193-224, 2007.

[7] J. Escher and G. Simonett. Classical solutions for Hele-Shaw models with surface tension. Adv. Differential Equations, 2:619-642, 1997.

[8] J. Escher and G. Simonett. A center manifold analysis for the Mullins-Sekerka model. J. Differential Equations, 143:267-292, 1998.

[9] M. Geissert, H. Heck, and M. Hieber. On the equation div $u=g$ and Bogovskiu's operator in Sobolev spaces of negative order. Oper. Theory Adv. Appl., 168:113-121, 2006.

[10] G. Grubb. Parameter-elliptic and parabolic pseudodifferential boundary problems in global $L_{p}$-spaces. Math. Z., 218:43-90, 1995.

[11] S. Hildebrandt. Analysis 2. Springer, Berlin - Heidelberg, 2003.

[12] C. Kneisel. Über das Stefan-Problem mit Oberflächenspannung und thermischer Unterkühlung. Hannover, Univ., Diss., 2007. Access: June 1, 2017.

[13] M. Köhne, J. Prüss, and M. Wilke. On quasilinear parabolic evolution equations in weighted $L^{p}$-spaces. J. Evol. Equ., 10:443-463, 2010.

[14] M. Köhne, J. Prüss, and M. Wilke. Qualitative behaviour of solutions for the two-phase Navier-Stokes equations with surface tension. Math. Ann., 356:737-792, 2013.

[15] L. D. Landau and E. M. Lifshitz. Fluid Mechanics. Pergamon Books Ltd., 1987.

[16] C. Liu, N. Sato, and Y. Tonegawa. Two-phase flow problem coupled with mean curvature flow. Interfaces Free Bound., 14(2):185-203, 2012. 


\section{B. REFERENCES}

[17] A. Lunardi. Interpolation Theory. Scuola Normale Superiore Pisa, 2. edition edition, 2009.

[18] J. Prüss and G. Simonett. Moving interfaces and quasilinear parabolic evolution equations. Monographs in Mathematics 105. Springer Switzerland, 2016.

[19] Y. Shibata and S. Shimizu. Maximal $L_{p}-L_{q}$ regularity for the two-phase Stokes equations; model problems. J. Differential Equations, 251:373-419, 2011.

[20] S. Shimizu. Maximal regularity and viscous incompressible flows with free interface, parabolic and Navier-Stokes equations. part 2. Banach Center Publ., Polish Acad. Sci. Inst. Math., Warsaw, 81:471-480, 2008.

[21] J. Simon. Sobolev, Besov, Nikol'siu fractional spaces: Imbeddings and comparisons for vector valued spaces on an interval. Ann. Mat. Pura Appl., 157:117-148, 1990.

[22] H. Triebel. Interpolation Theory, Function Spaces, Differential Operators. NorthHolland Publishing Company, Amsterdam - New York - Oxford, 1978.

[23] H. Triebel. Theory of Function Spaces. Monographs in Mathematics 78. Birkhäuser, Basel, 1983. 